\title{
GW Orionis: Inner disk readjustments in a triple system ${ }^{\star}$
}

\author{
M. Fang ${ }^{1}$, A. Sicilia-Aguilar ${ }^{2,1}$, V. Roccatagliata ${ }^{3}$, D. Fedele ${ }^{4}$, Th. Henning ${ }^{5}$, C. Eiroa ${ }^{1}$, and A. Müller ${ }^{6}$ \\ ${ }^{1}$ Departamento de Física Teórica, Universidad Autónoma de Madrid, 28049 Cantoblanco, Madrid, Spain \\ e-mail: mfang.cn@gmail.com \\ 2 SUPA, School of Physics and Astronomy, University of St Andrews, North Haugh, St Andrews KY16 9SS, UK \\ 3 Universitäts-Sternwarte München, Ludwig-Maximilians-Universität, Scheinerstr. 1, 81679 München, Germany \\ ${ }^{4}$ Max-Planck Institut für Extraterrestrische Physik, Giessenbachstrasse 1, 85748 Garching, Germany \\ 5 Max-Planck Institut für Astronomie, Königstuhl 17, 69117 Heidelberg, Germany \\ ${ }^{6}$ European Southern Observatory, Alonso de Cordova 3107, Casilla 19001 Vitacura, Santiago, Chile
}

Received 6 May 2014 / Accepted 3 July 2014

\begin{abstract}
Context. Disks are expected to dissipate quickly in binary or multiple systems. Investigating such systems can improve our knowledge of the disk dispersal. The triple system GW Ori, still harboring a massive disk, is an excellent target.

Aims. We study the young stellar system GW Ori, concentrating on its accretion, wind activity and disk properties.

Methods. We use high-resolution optical spectra of GW Ori to do spectral classification and derive the radial velocities (RV). We analyze the wind and accretion activity using the emission lines in the spectra. We also use $U$-band photometry, which has been collected from the literature, to study the accretion variability of GW Ori. We characterize the disk properties of GW Ori by modeling its spectral energy distribution (SED).

Results. By comparing our data to the synthetical spectra, we classify GW Ori as a G8 star. Based on the RVs derived from the optical spectra, we confirm the previous result as a close companion in GW Ori with a period of $\sim 242$ days and an orbital semi-major axis of $\sim 1$ AU. The RV residuals after the subtraction of the orbital solution with the equivalent widths (EW) of accretion-related emission lines vary with periods of 5-6.7 days during short-time intervals, which are caused by the rotational modulation. The $\mathrm{H} \alpha$ and $\mathrm{H} \beta$ line profiles of GW Ori can be decomposed in two central-peaked emission components and one blue-shifted absorption component. The blue-shifted absorption components are due to a disk wind modulated by the orbital motion of the close companion. Therefore, the systems like GW Ori can be used to study the extent of disk winds. We find that the accretion rates of GW Ori are rather constant but can occasionally be enhanced by a factor of 2-3. We reproduce the SED of GW Ori by using disk models with gaps $\sim 25-55$ AU in size. A small population of tiny dust particles within the gap produces the excess emission at near-infrared bands and the strong and sharp silicate feature at $10 \mu \mathrm{m}$. The SED of GW Ori exhibits dramatic changes on timescales of $\sim 20 \mathrm{yr}$ in the near-infrared bands, which can be explained as the change in the amount and distribution of small dust grains in the gap. We collect a sample of binary/multiple systems with disks in the literature and find a strong positive correlation between their gap sizes and separations from the primaries to companions, which is generally consistent with the prediction from the theory.
\end{abstract}

Key words. stars: pre-main sequence - binaries: spectroscopic - stars: individual: GW Orionis - accretion, accretion disks line: profiles

\section{Introduction}

Young stars are born with disks as a result of angular momentum conservation (Shu 1977). Observations suggest disk lifetimes of a few Myrs (Strom et al. 1989; Haisch et al. 2001; Hillenbrand 2002; Hernández et al. 2007; Sicilia-Aguilar et al. 2006; Fedele et al. 2010; Fang et al. 2012, 2013b). However, the physical processes in the disk evolution are still poorly understood. Spectroscopic and/or imaging surveys toward nearby field dwarfs suggest that 50\% of G-type stars have companions (Duquennoy \& Mayor 1991), and 30-40\% of M-type stars are in binary/multiple systems (Fischer \& Marcy 1992; Janson et al. 2012). The fractions of binary/multiple systems are even higher in star-forming regions (Ghez et al. 1993, 1997; Leinert et al. 1993; Lafrenière et al. 2008; Kraus et al. 2011). Therefore, the interaction between disks and companions has been proposed as an efficient mechanism to dissipate disks (Lin \& Papaloizou 1993). Observationally, this mechanism can be very efficient in disk disperal at very early stages ( $<1 \mathrm{Myr}$, Cieza et al. 2009; Kraus et al. 2012) and may play a key role in dissipating the

* Table 4 is available in electronic form at http: //www. aanda.org disks in sparse stellar associations (Bouwman et al. 2006; Fang et al. 2013b).

In a young binary system, two types of disks could be present: a circumstellar disk surrounding each star in the system and a circumbinary disk around the binary pair (Lubow \& Artymowicz 2000). Circumstellar disks can be truncated outside by the tidal companion-disk interaction and can be misaligned, while circumbinary disks can be carved out inside by the companions (Lin \& Papaloizou 1993; Artymowicz \& Lubow 1994; Roccatagliata et al. 2011). Gaps, quickly produced in the inner regions of circumbinary disks, can separate circumstellar disks and circumbinary disks (Lin \& Papaloizou 1993; Lubow $\&$ Artymowicz 2000). Such gaps in circumbinary disks, as suggested in the spectral energy distributions (SED) of some binary systems (Jensen \& Mathieu 1997), have been directly detected with millimeter interferometry (Rosenfeld et al. 2013; Isella et al. 2013).

For a circumbinary disk with a disk thickness-to-radius ratio $h / r>0.05$, the simulations indicate that the gap in the inner disk region can be replenished with material from the circumbinary disk in the form of gas streams, which can supply the mass for 
Table 1. Observing log for the spectroscopy.

\begin{tabular}{|c|c|c|c|c|c|c|c|c|c|}
\hline ID & Obs. Date & JD-2 450000 & Instrument & Exp. time (s) & ID & Obs. Date & JD-2 450000 & Instrument & Exp. time (s) \\
\hline 1 & 2007-11-08 & 4412.689 & FEROS & 900.0 & 30 & 2009-01-13 & 4844.538 & FEROS & 960.0 \\
\hline 2 & 2007-11-09 & 4413.727 & FEROS & 1300.0 & 31 & 2009-01-13 & 4844.680 & FEROS & 960.0 \\
\hline 3 & 2008-01-31 & 4496.530 & FEROS & 1200.0 & 32 & 2009-01-14 & 4845.606 & FEROS & 960.0 \\
\hline 4 & 2008-11-07 & 4777.866 & FEROS & 960.0 & 33 & 2009-02-12 & 4874.520 & HARPS & 540.0 \\
\hline 5 & 2008-11-09 & 4779.777 & FEROS & 900.0 & 34 & 2009-02-13 & 4875.532 & HARPS & 540.0 \\
\hline 6 & 2008-11-09 & 4779.788 & FEROS & 900.0 & 35 & 2009-02-14 & 4876.574 & HARPS & 540.0 \\
\hline 7 & 2008-11-11 & 4781.869 & FEROS & 900.0 & 36 & 2009-02-15 & 4877.543 & HARPS & 540.0 \\
\hline 8 & 2008-11-12 & 4782.849 & FEROS & 900.0 & 37 & 2009-03-01 & 4891.508 & HARPS & 600.0 \\
\hline 9 & 2008-11-13 & 4783.851 & FEROS & 720.0 & 38 & 2009-03-01 & 4892.501 & HARPS & 600.0 \\
\hline 10 & 2008-11-14 & 4784.690 & FEROS & 720.0 & 39 & 2009-03-03 & 4893.519 & HARPS & 600.0 \\
\hline 11 & 2008-11-16 & 4786.777 & HARPS & 1200.0 & 40 & 2009-03-04 & 4894.565 & HARPS & 600.0 \\
\hline 12 & 2008-11-17 & 4787.747 & HARPS & 1200.0 & 41 & 2009-04-28 & 4950.469 & FEROS & 960.0 \\
\hline 13 & 2008-11-18 & 4788.813 & HARPS & 900.0 & 42 & 2009-10-01 & 5105.810 & FEROS & 465.8 \\
\hline 14 & 2009-01-05 & 4836.533 & FEROS & 960.0 & 43 & 2009-10-02 & 5106.849 & FEROS & 960.0 \\
\hline 15 & 2009-01-05 & 4836.629 & FEROS & 960.0 & 44 & 2009-10-04 & 5108.867 & FEROS & 1200.0 \\
\hline 16 & 2009-01-06 & 4837.538 & FEROS & 960.0 & 45 & 2009-10-07 & 5111.876 & FEROS & 960.0 \\
\hline 17 & 2009-01-06 & 4837.599 & FEROS & 960.0 & 46 & 2009-12-14 & 5179.775 & FEROS & 900.0 \\
\hline 18 & 2009-01-07 & 4838.538 & FEROS & 960.0 & 47 & 2009-12-15 & 5180.777 & FEROS & 900.0 \\
\hline 19 & 2009-01-07 & 4838.689 & FEROS & 960.0 & 48 & 2009-12-16 & 5181.762 & FEROS & 900.0 \\
\hline 20 & 2009-01-08 & 4839.567 & FEROS & 960.0 & 49 & 2010-01-03 & 5199.705 & FEROS & 960.0 \\
\hline 21 & 2009-01-08 & 4839.720 & FEROS & 960.0 & 50 & 2010-01-06 & 5202.702 & FEROS & 960.0 \\
\hline 22 & 2009-01-09 & 4840.560 & FEROS & 960.0 & 51 & $2010-01-28$ & 5224.650 & FEROS & 465.8 \\
\hline 23 & 2009-01-09 & 4840.687 & FEROS & 960.0 & 52 & $2010-01-28$ & 5224.663 & FEROS & 1200.0 \\
\hline 24 & 2009-01-10 & 4841.542 & FEROS & 960.0 & 53 & $2010-01-29$ & 5225.664 & FEROS & 900.0 \\
\hline 25 & 2009-01-10 & 4841.632 & FEROS & 960.0 & 54 & 2010-01-30 & 5226.644 & FEROS & 900.0 \\
\hline 26 & 2009-01-11 & 4842.572 & FEROS & 960.0 & 55 & $2010-02-27$ & 5254.566 & FEROS & 900.0 \\
\hline 27 & 2009-01-11 & 4842.633 & FEROS & 960.0 & 56 & 2010-03-05 & 5260.595 & FEROS & 900.0 \\
\hline 28 & 2009-01-12 & 4843.538 & FEROS & 960.0 & 57 & 2010-03-13 & 5268.535 & FEROS & 900.0 \\
\hline 29 & 2009-01-12 & 4843.619 & FEROS & 960.0 & 58 & 2010-03-14 & 5269.541 & FEROS & 900.0 \\
\hline
\end{tabular}

accretion onto the central binary (Artymowicz \& Lubow 1996). The simulations also find that the periodic perturbations in the circumstellar disk caused by the orbital motion of the close companion can induce mass flow across the gap, resulting in accretion that changes with the orbital motion of binary. For a binary with a high eccentricity $(e=0.5)$ and a mass ratio near one, the accretion rate of the system can be strongly modulated in time and reach a maximum near periastron. However, when the eccentricity of a binary is lower $(e=0.1)$, the accretion rate of the system can be still pulsed with the orbital motions, but the enhancement can be smooth and less notable than those of the high- $e$ cases (Artymowicz \& Lubow 1996). The scenario of pulsed accretion in binary systems, as predicted by the simulations, is confirmed by the observations of only few cases, such as DQ Tau and UZ Tau E (Basri et al. 1997; Martín et al. 2005; Jensen et al. 2007). An investigation of other binaries can contribute in understanding the accretion processes in such systems.

The object GW Ori, located at $\lambda$ Ori $(\sim 400 \mathrm{pc}$, Murdin $\&$ Penston 1977), was first revealed as a spectroscopic binary (GW Ori A/B) with an orbital period of $\sim 242$ days and a separation of $\sim 1 \mathrm{AU}$ (Mathieu et al. 1991). The new nearinfrared interferometric observations confirm the existence of a close companion (GW Ori B) and detect a second (GW Ori C) with a projected separation of $\sim 8 \mathrm{AU}$ from GW Ori A (Berger et al. 2011). Though it is a triple system, observations show that GW Ori is still harboring a massive disk $\left(\sim 0.3 M_{\odot}\right)$ with a high accretion rate of $\sim 3 \times 10^{-7} M_{\odot} \mathrm{yr}^{-1}$ (Mathieu et al. 1995; Calvet et al. 2004). Thus, GW Ori is a very interesting target and deserves further detailed investigation.

We obtain 58 high-resolution optical spectra of GW Ori. As a complement, we also collect a large set of archive data, including a set of multi-epoch broad band photometry in different bands, and the infrared spectrum from the Spitzer InfraRed Spectrograph (IRS, Houck et al. 2004). Using the accretionrelated emission lines in the optical spectra and $U$-band photometry, we investigate the accretion behavior of GW Ori, which concentrates mainly on the accretion variability that has not been studied in detail yet. With the infrared data, we will characterize the disk properties of GW Ori. We arrange this paper as follows. In Sect. 2, we describe the observations and reduction of the optical data. In Sect. 3, we present our results which are then discussed in Sect. 4. We summarize our results in Sect. 5.

\section{Observations and data reduction}

Our optical spectra of GW Ori were taken with the Fiber-fed Extended Range Optical Spectrograph (FEROS, Kaufer et al. 1999), which is mounted on the $2.2 \mathrm{~m}$ MPG/ESO (Max-Planck Gesellschaft/European Southern Observatory) telescope, and the High Accuracy Radial velocity Planet Searcher (HARPS, Mayor et al. 2003), which is mounted on the $3.6 \mathrm{~m}$ telescope. Both telescopes are located at La Silla Observatory. The FEROS has a spectral resolution of $\lambda / \Delta \lambda \sim 48000$ with a wavelength coverage of 3600-9200 $\AA$. The HARPS has a higher spectral resolution $(\lambda / \Delta \lambda \sim 115000)$, but a narrower wavelength coverage (3800-6900 $\AA$ ). With the two instruments, a total of 58 spectra were obtained during 2007-2010 (see Table 1 for a detailed description of these observations). Both FEROS and HARPS have their own online data reduction pipelines, which can automatically produce science-quality spectra with calibrated wavelengths from observational raw data. Recently, Müller et al. (2013) found that the barycentric correction of the FEROS data reduction pipeline is inaccurate, as it induces an artificial one-year period with a semi-amplitude of $62 \mathrm{~m} \mathrm{~s}^{-1}$. Following 

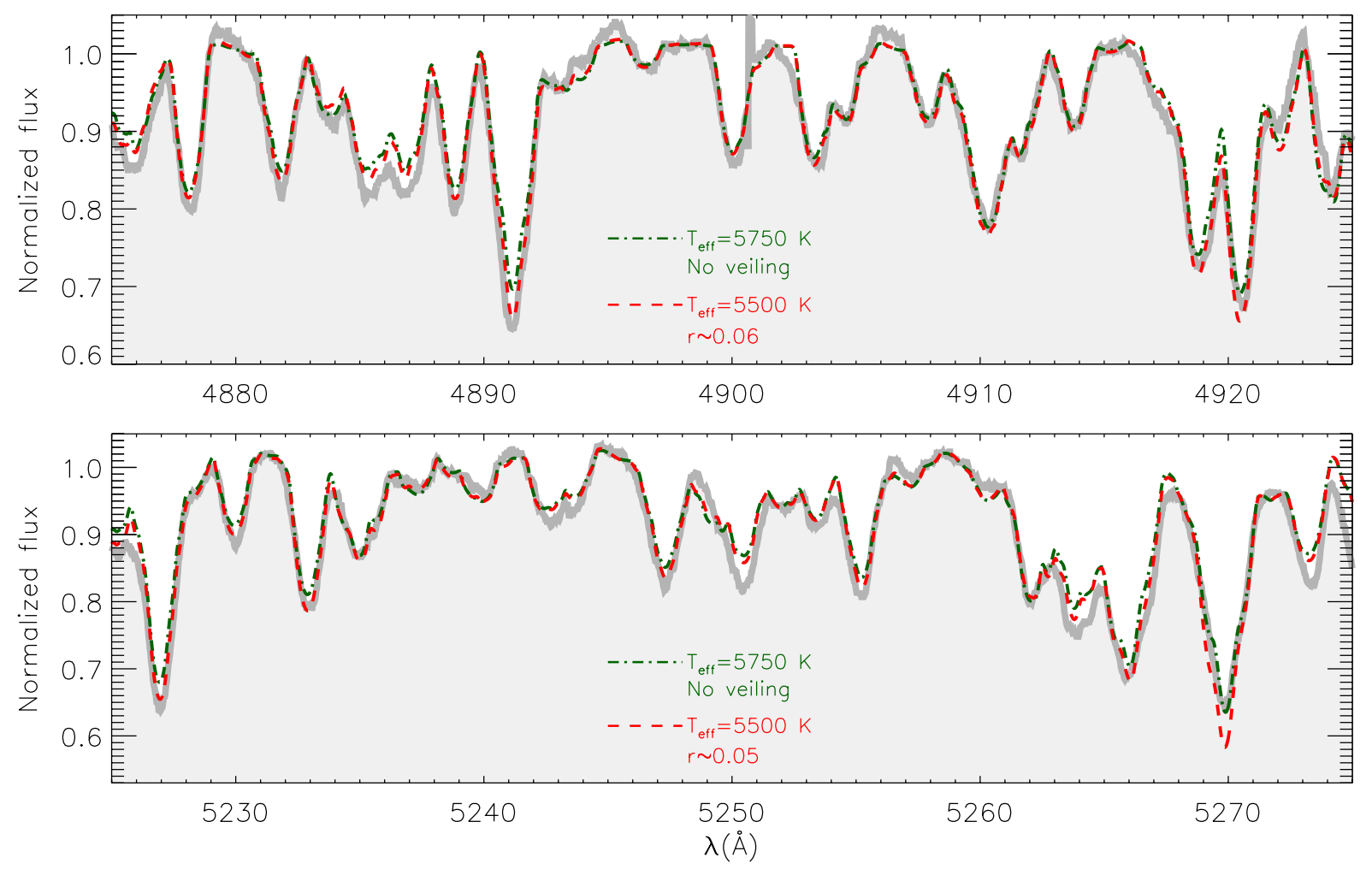

Fig. 1. Comparison of the observed spectrum (thick gray lines) of GW Ori, which is combined by using the data observed during Jan. 6-11 in 2009 , and synthetical spectra at $T_{\text {eff }}=5750$ (the dash-dotted lines) and $5500 \mathrm{~K}$ (the dashed lines). The spectra are all normalized. The best veiling value $(r)$ is also given for each model spectrum.

Müller et al. (2013), we apply a more precise barycentric correction that is calculated with the IDL code "baryvel.pro", which is based on the method in Stumpff (1980) and gives an accuracy of $\sim 1 \mathrm{~m} \mathrm{~s}^{-1}$. The corrected FEROS spectra are then used in our analysis.

\section{Results}

\subsection{Stellar properties}

In the literature, the spectral type of GW Ori ranges from K3 to G0 (Joy 1949; Herbig 1977; Heckmann 1975; Calvet et al. 2004; Weise et al. 2010). We classify GW Ori by comparing its observed spectra with synthetical spectra. To improve the quality of the observational data, we combine the FEROS spectra $^{1}$, which is observed during Jan. 6-11 in 2009, to one spectrum. With these data, we do not see any obvious variations in photospheric absorption features among the individual spectra. For comparison, we used synthetical spectra extracted from Coelho et al. (2005) with a solar abundance and a surface gravity $\log g=3.5^{2}$. The comparison spectra were first degraded to match the spectral resolution of FEROS and then rotationally broadened with a rotation velocity $v \sin i_{*}=43.7 \mathrm{~km} \mathrm{~s}^{-1}$ (Weise et al. 2010).

\footnotetext{
1 Before the combination, each spectrum used has been corrected for the Doppler shift by using the value derived in Sect. 3.2 and normalized to avoid the possible variations in the shape of the spectra.

2 Using the stellar masses and radius of GW Ori from Calvet et al. (2004), the surface gravity of GW Ori is estimated to be $\log g \sim 3.3$. Therefore, for simplicity, we only select the synthetical spectra with $\log g=3.5$ from Coelho et al. (2005).
}

The accretion shock can produce excess continuum emissions, which fill the photospheric absorption features and induce a veiling effect on the spectra (Calvet \& Gullbring 1998). According to Calvet et al. (2004), the accretion luminosity of GW Ori is $\sim 8 \%$ of its stellar luminosity. In addition, the excess continuum emission peaks at the UV band. Therefore, veiling is expected to be insignificant for GW Ori in optical bands. However, we still include veilling in the spectral comparison as an independent check. We divide spectra into different wavelength bins with a size of $50 \AA$. Within each bin, we include a free parameter $r$ to simulate veiling in a way similar to that in Hartigan et al. (1989). The $r$ value is set to be constant within one wavelength bin and can be optimized by minimizing $\chi^{2}$, as defined by $\chi^{2}=\sum\left(F_{\mathrm{Obs}, \lambda}-\frac{F_{\mathrm{Syn}, \lambda}+r}{1+r}\right)^{2}$, where $F_{\mathrm{Obs}, \lambda}$ is the flux of the observed spectrum at the wavelength $\lambda$, and $F_{\text {Syn, } \lambda}$ is the flux of the theoretical spectrum at the corresponding wavelength. We find that the GW Ori spectrum can be best reproduced by synthetical spectra with effective temperatures $\left(T_{\text {eff }}\right)$ between 5500 and $5750 \mathrm{~K}$ (closer to $5500 \mathrm{~K}$ ) and a negligible veiling $(r<0.1)$, which is consistent with that suggested by Calvet et al. (2004). In Fig. 1, we show comparisons of the spectra as an example within the wavelength ranges of 4875-4925 and $5225-5275 \AA$. Hereafter, we take $5500 \mathrm{~K}$ as the effective temperature of GW Ori, which corresponds to a spectral type $\sim \mathrm{G} 8$, according to the relation between spectral types and $T_{\text {eff }}$ that is given in Kenyon \& Hartmann (1995).

Using our derived spectral type with photometric data in Calvet et al. (2004), we calculate the visual extinction $\left(A_{V}\right)$ and bolometric luminosity $\left(L_{\star}\right)$ of the primary of GW Ori (GW Ori A), assuming that the primary dominates the emission of the system. In the calculation, we use the method described 
Table 2. Relative radial velocity of GW Ori.

\begin{tabular}{|c|c|c|c|c|c|c|c|c|c|}
\hline ID & JD-2 450000 & $\begin{array}{l}\text { Rel. RV } \\
\left(\mathrm{km} \mathrm{s}^{-1}\right)\end{array}$ & $\begin{array}{c}\sigma \text { (Rel. RV) } \\
\left(\mathrm{km} \mathrm{s}^{-1}\right)\end{array}$ & $\begin{array}{c}(\mathrm{O}-\mathrm{C}) \\
\left(\mathrm{km} \mathrm{s}^{-1}\right)\end{array}$ & ID & JD-2 450000 & $\begin{array}{l}\text { Rel. RV } \\
\left(\mathrm{km} \mathrm{s}^{-1}\right)\end{array}$ & $\begin{array}{c}\sigma(\text { Rel. RV }) \\
\left(\mathrm{km} \mathrm{s}^{-1}\right)\end{array}$ & $\begin{array}{c}(\mathrm{O}-\mathrm{C}) \\
\left(\mathrm{km} \mathrm{s}^{-1}\right) \\
\end{array}$ \\
\hline 1 & 4412.689 & +5.17 & 0.12 & +1.89 & 30 & 4844.538 & +5.39 & 0.09 & +0.56 \\
\hline 2 & 4413.727 & +4.63 & 0.07 & +1.40 & 31 & 4844.680 & +4.90 & 0.09 & +0.08 \\
\hline 3 & 4496.530 & +0.18 & 0.08 & +2.01 & 32 & 4845.606 & +4.73 & 0.07 & -0.09 \\
\hline 4 & 4777.866 & +0.09 & 0.06 & +0.21 & 33 & 4874.520 & +6.03 & 0.07 & +1.56 \\
\hline 5 & 4779.777 & -0.20 & 0.06 & -0.30 & 34 & 4875.532 & +4.30 & 0.06 & -0.13 \\
\hline $6^{a}$ & 4779.788 & +0.00 & $0.06^{b}$ & -0.10 & 35 & 4876.574 & +4.61 & 0.06 & +0.21 \\
\hline 7 & 4781.869 & +0.27 & 0.06 & -0.09 & 36 & 4877.543 & +3.11 & 0.06 & -1.25 \\
\hline 8 & 4782.849 & +0.98 & 0.06 & +0.50 & 37 & 4891.508 & +3.40 & 0.06 & -0.35 \\
\hline 9 & 4783.851 & +1.12 & 0.06 & +0.52 & 38 & 4892.501 & +4.88 & 0.07 & +1.18 \\
\hline 10 & 4784.690 & +0.94 & 0.07 & +0.23 & 39 & 4893.519 & +3.31 & 0.06 & -0.34 \\
\hline 11 & 4786.777 & +2.03 & 0.06 & +0.83 & 40 & 4894.565 & +3.18 & 0.06 & -0.41 \\
\hline 12 & 4787.747 & +0.76 & 0.07 & -0.56 & 41 & 4950.469 & +0.03 & 0.06 & +0.24 \\
\hline 13 & 4788.813 & +1.70 & 0.07 & +0.24 & 42 & 5105.810 & +3.61 & 0.25 & -0.95 \\
\hline 14 & 4836.533 & +4.59 & 0.06 & -0.16 & 43 & 5106.849 & +4.96 & 0.18 & +0.43 \\
\hline 15 & 4836.629 & +4.83 & 0.10 & +0.07 & 44 & 5108.867 & +4.43 & 0.12 & -0.04 \\
\hline 16 & 4837.538 & +4.61 & 0.06 & -0.17 & 45 & 5111.876 & +4.00 & 0.09 & -0.38 \\
\hline 17 & 4837.599 & +4.63 & 0.07 & -0.14 & 46 & 5179.775 & +0.58 & 0.06 & -0.06 \\
\hline 18 & 4838.538 & +5.03 & 0.07 & +0.24 & 47 & 5180.777 & -0.62 & 0.06 & -1.20 \\
\hline 19 & 4838.689 & +4.96 & 0.11 & +0.17 & 48 & 5181.762 & +0.22 & 0.06 & -0.29 \\
\hline 20 & 4839.567 & +4.63 & 0.08 & -0.17 & 49 & 5199.705 & -0.85 & 0.06 & -0.13 \\
\hline 21 & 4839.720 & +5.25 & 0.08 & +0.45 & 50 & 5202.702 & -0.05 & 0.06 & +0.86 \\
\hline 22 & 4840.560 & +4.98 & 0.08 & +0.17 & 51 & 5224.650 & -3.04 & 0.14 & -1.13 \\
\hline 23 & 4840.687 & +5.01 & 0.07 & +0.20 & 52 & 5224.663 & -3.00 & 0.07 & -1.08 \\
\hline 24 & 4841.542 & +4.79 & 0.08 & -0.02 & 53 & 5225.664 & -2.03 & 0.13 & -0.10 \\
\hline 25 & 4841.632 & +4.75 & 0.07 & -0.06 & 54 & 5226.644 & -1.89 & 0.09 & +0.06 \\
\hline 26 & 4842.572 & +4.40 & 0.10 & -0.42 & 55 & 5254.566 & -1.14 & 0.06 & -0.32 \\
\hline 27 & 4842.633 & +4.36 & 0.06 & -0.46 & 56 & 5260.595 & -0.46 & 0.06 & -0.28 \\
\hline 28 & 4843.538 & +4.70 & 0.09 & -0.12 & 57 & 5268.535 & -0.56 & 0.10 & -1.35 \\
\hline 29 & 4843.619 & +4.85 & 0.08 & +0.03 & 58 & 5269.541 & +0.03 & 0.10 & -0.88 \\
\hline
\end{tabular}

Notes. Column 3: RVs relative to the ID 6 spectrum. Column 5: RV residuals from the orbital solution. a: Template used for deriving the relative RVs of other spectra. b: Typical error for other spectra.

in Fang et al. (2009) by adopting a total to selective extinction value typical of interstellar medium dust $\left(R_{V}=3.1\right)$ and the extinction law from Cardelli et al. (1989). The resulting $A_{V}$ and $L_{\star}$ of GW Ori A are $1.5 \pm 0.1 \mathrm{mag}$ and $48 \pm 10 L_{\odot}$, respectively. We derive the stellar mass $\left(M_{\star}\right)$ and age of GW Ori A using the three sets of publicly available pre-main sequence (PMS) evolutionary tracks from Siess et al. (2000; S00), Dotter et al. (2008; D08), and Tognelli et al. (2011; Pisa11). The masses and ages of GW Ori A are $3.7 \pm 0.3 M_{\odot}$ and $0.9 \pm 0.3 \mathrm{Myr}$ from S00, $4.0 \pm 0.2 M_{\odot}$ and $0.4 \pm 0.1 \mathrm{Myr}$ from D08, and 3.9 $\pm 0.2 M_{\odot}$ and $0.7 \pm 0.2 \mathrm{Myr}$ from Pisa11. In the following, we use the weighted mean, 3.9 $\pm 0.2 M_{\odot}$, as the mass of GW Ori A. With such a high mass, GW Ori A would be a B6 main-sequence star (Schaller et al. 1992). The late spectral type (G8) of GW Ori A suggests that it is at an evolutionary stage earlier than Herbig Be stars. According to the three PMS evolutionary tracks for a young star with a mass of $4 M_{\odot}$, there is a rapid transition from $G$ type to B type at an age of $\sim 1 \mathrm{Myr}$, which may explain why there are so few known young stars, like GW Ori A, with such high masses but with late spectral types.

\subsection{Radial velocity, Keplerian orbital solution, and rotation}

We derive the radial velocity (RV) of each spectrum of GW Ori using the cross-correlation method by taking one observed spectrum (ID 6 in Table 1) as the template. The relative RVs are listed in Table 2. In Fig. 2a, we show the generalized Lomb-Scargle (GLS) periodogram of the RVs that are calculated using the

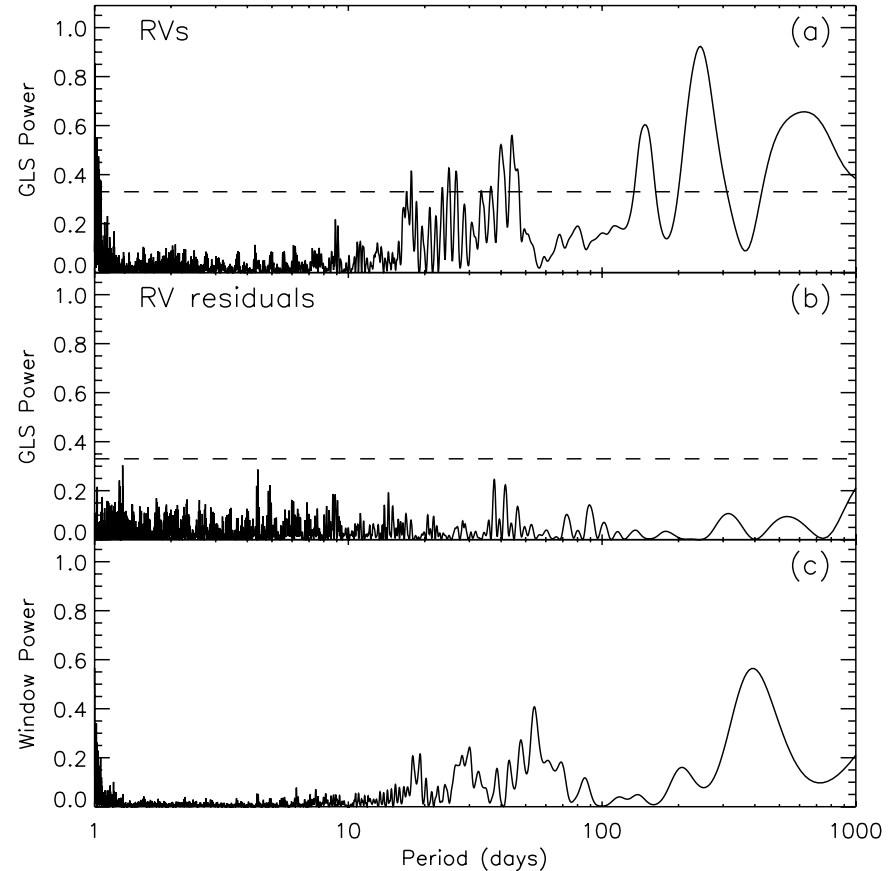

Fig. 2. a) Generalized Lomb-Scargle periodogram of the RV measurements of GW Ori. b) Generalized Lomb-Scargle periodogram for the RV residuals after subtracting a one-companion fit. c) Window function. The dashed lines in panels a), b) indicate the power level for an FAP of 0.01 , as computed by GLS. 


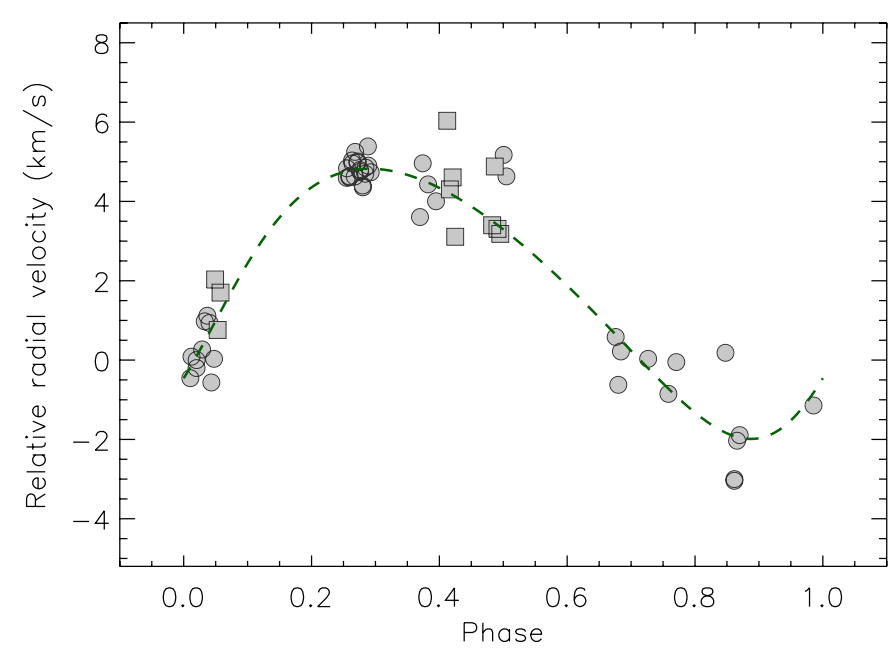

Fig. 3. Phase-folded relative RV curve (dash line) of GW Ori. The filled circles show our measurements of the spectra from FEROS, and the filled boxes are for the data from HARPS.

Table 3. Orbital elements for GW Ori.

\begin{tabular}{ccc}
\hline \hline Parameters & This work & In Mathieu et al. (1991) \\
$P($ days $)$ & $241.6 \pm 1.5$ & $241.9 \pm 1.0$ \\
$T_{\mathrm{p}}$ & $4774.9 \pm 8.0$ & \\
$(\mathrm{JD}-2450000)$ & & \\
$\mathrm{e}$ & $0.18 \pm 0.06$ & $0.04 \pm 0.06$ \\
$\omega(\mathrm{deg})$ & $236.6 \pm 13.4$ & $71 \pm 60$ \\
$K\left(\mathrm{~km} \mathrm{~s}^{-1}\right)$ & $3.41 \pm 0.17$ & $4.7 \pm 0.3$ \\
$f(m)\left(M_{\odot}\right)$ & $(9.39 \pm 1.16) \times 10^{-4}$ & $2.6 \pm 0.5 \times 10^{-3}$ \\
$\sigma(\mathrm{O}-\mathrm{C})\left(\mathrm{km} \mathrm{s}^{-1}\right)$ & 0.7 & 1.3 \\
\hline With $m_{1}=3.9 \pm 0.2 M_{\odot}$ & & \\
$m_{2} \sin i\left(M_{\odot}\right)$ & $0.25 \pm 0.01$ & \\
$a_{2}(\mathrm{AU})$ & $1.20 \pm 0.02$ & \\
\hline
\end{tabular}

method in Zechmeister \& Kürster (2009). The GLS periodogram shows three strong peaks at $\sim 120,240$, and 600 days, respectively. The strongest peak is at $\sim 240$ days with a false-alarm probability (FAP) of $1.7 \times 10^{-28}$ estimated by GLS.

We achieve a one-companion Keplerian orbital solution for GW Ori by using the fitting procedure in Wright \& Howard (2009) and estimate the uncertainties of parameters by using the bootstrapping routines in Wang et al. (2012). A phase-folded RV curve of GW Ori is shown in Fig. 3. The best-fit orbital parameters are summarized in Table 3, which includes the orbital period $P$, a Julian date of periastron passage $T_{\mathrm{P}}$, the eccentricity $e$, the periastron angle $\omega$, the semi-amplitude of the RV curve $K$, the mass function $f(m)$, and the rms residual velocities from the orbital solution. In Table 3, we also present the orbital parameters from Mathieu et al. (1991) as a comparison. We note our rms residual velocity is about half of that in Mathieu et al. (1991). Both works agree with each other on the orbital periods. Our eccentricity is marginally larger than the previous one. The largest differences between both works arise mostly in $\omega, K$, and $f(m)$. According to the uncertainties of $\omega$, our data may provide a better constraint on $\omega$ than Mathieu et al. (1991). Taking $3.9 \pm 0.2 M_{\odot}$ as the mass of GW Ori A, we obtain a minimum companion mass $\left(m_{2} \sin i\right) \sim 0.25 M_{\odot}$, and an orbital semi-major axis $(a)$ of $1.20 \mathrm{AU}$. The $a$ value is consistent with the one in Mathieu et al. (1991).

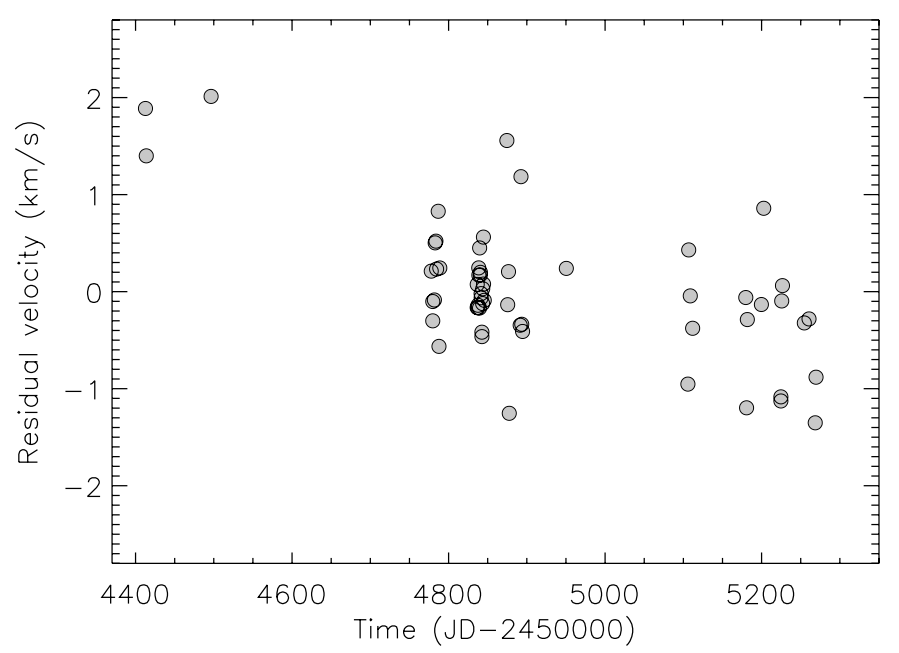

Fig. 4. Residual velocities of GW Ori plotted as a function of observational dates.

Table 2 lists the individual RV residuals from the orbital solution. The rms residual velocity is $\sim 0.7 \mathrm{~km} \mathrm{~s}^{-1}$, which considerably higher than internal measurement errors $\left(\lesssim 0.1 \mathrm{~km} \mathrm{~s}^{-1}\right)$. We show the GLS periodogram of the RV residuals in Fig. 2b. We note that the two peaks (120 and 600 days) shown in Fig. 2a disappear, and no significant periods with FAP $<0.01$ are present in the periodogram. In Fig. 4, we show the RV residuals with respect to observational dates and note a systematic shift in the RV residuals with the time. Mathieu et al. (1991) also found a systematic shift in their RV residuals and explained it as being caused by a second companion (GW Ori C). The GW Ori C has been confirmed with the infrared interferometric technique by Berger et al. (2011) and is located at a project separation $\sim 8$ AU from GW Ori A, indicating an orbital period $\sim 3600$ days. Our observations only have a time span of $\sim 850$ days and, thus, cannot provide any useful constrains on the orbital parameters of GW Ori C.

In our datasets, there are two time intervals, Nov. 7-18, 2008 and Jan. 5-14, 2009, with very dense time coverage. In Figs. 5 and 6, we show the RV residuals during the two periods and note periodic variations in the RV residuals. We fit the data points $\left(v_{\text {res }}\right)^{3}$ by using the form $v_{\text {res }}=v_{\max } \times \sin \left(\frac{2 \pi t}{\tau}+\theta\right)+v_{0}$, where $v_{0}$ is the systematic shift caused by the second companion, and $\tau$ is the period of the $v_{\text {res }}$ variation. The best-fit results with $\tau \sim 6.7$ and 5.0 days are also shown in Fig. 5 and 6, respectively. We calculate the GLS periodograms of the RV residuals for the data taken during the two time intervals and show them in Figs. 7a, b. The peaks of $\sim 6.7$ and 5.0 can be noted in the two periodograms with FAP $\sim 0.07$ and 0.01 , respectively. In Fig. 7c, we show the GLS periodogram for the data taken between Nov. 7, 2008 and Apr. 28, 2009. We only use the data during that time because the systematic shifts caused by the second companion are small (see Fig. 4). In Fig. 7c, no strong peaks can be noted, suggesting the periodic variations shown in Figs. 5 and 6 are not stable. We also note that the best-fit functions for the two sets of data are inconsistent in both amplitudes and phases. Thus, the period of 5.0-6.7 days cannot be due to a new companion. One promising explanation could be rotational modulation (see e.g. Queloz et al. 2001; Prato et al. 2008; Müller et al. 2011). The disappearance

3 In each fit, we have excluded one data point: ID 11 in Fig. 5 and ID 20 in Fig. 6. The two data points seem to deviate very much from the global trends of the $v_{\text {res }}$ variations. The two data points are also excluded when we calculate the GLS periodograms in Figs. 7a, b. 


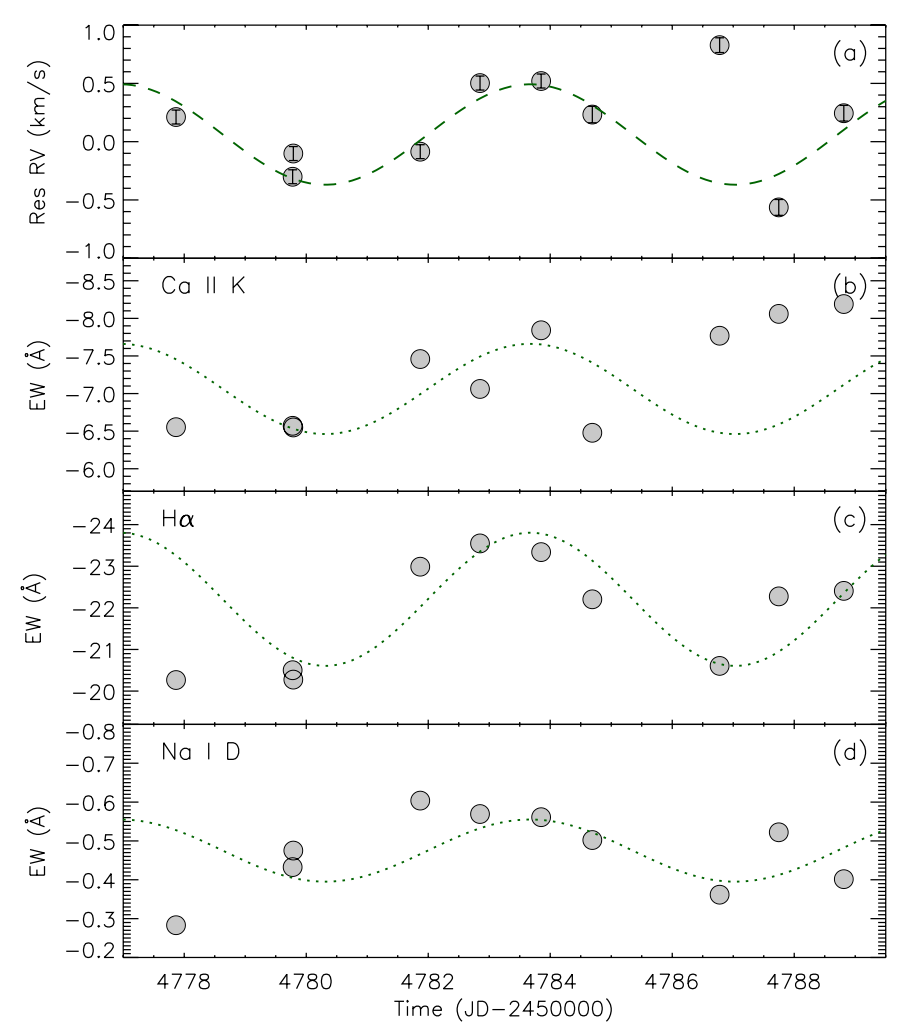

Fig. 5. a) Residual velocities of GW Ori. The error bars of individual measurements are shown. The dashed line is the fit to the residual RVs. The dotted lines in panels $\mathbf{b}), \mathbf{c})$, d) are sine functions with the fitted phase and period from panel a). We manually shift the sine functions and adjust the amplitudes to match the observed EWs of each emission line.

and appearance of the spots, as well as the variations of filling factors of spots on the stellar surface, can lead to the change in both amplitudes and phases in the periodic variations of the RV residuals due to the rotational modulation.

During these two time intervals, we find that the equivalent widths (EW) of accretion-related emission lines exhibit similar periodic variations to the RV residuals, although less distinct. Figures 5 and 6 show the EWs of three accretion-related emission lines, $\mathrm{Ca}$ II $\mathrm{K}$ line, $\mathrm{H} \alpha$, and $\mathrm{Na}$ I D line, with respect to the observational dates as examples (see Sect. 3.3 for a detail description of emission lines in the spectra of GW Ori). The periodic variation of the EWs of accretion-related emission lines can be also explained as a rotational modulation since the filling factor of the accretion-shock region on the stellar surface is small, and the accretion streams are usually not azimuthally symmetrically distributed with respect to the stellar rotational axis (Johns \& Basri 1995b; Romanova et al. 2004; Bouvier et al. 2007). In addition to the rotational modulation, the $E W$ variation of accretion-related emission lines can also be caused by accretion variation, such as non-steady accretion on the timescale of hours, the global instabilities of the magnetospheric structure on the timescale of months, or the pulsed accretion due to the orbital motion for binaries (Gullbring et al. 1996; Bouvier et al. 2003; Artymowicz \& Lubow 1996). These factors can contaminate the periodic behaviors of EWs of the emission lines in Figs. 5 and 6.

Taking the stellar radius $7.6 R_{\odot}$, the break-up velocity of GW Ori A is estimated to be $442 \mathrm{~km} \mathrm{~s}^{-1}$, and its lower-limit rotational period is 0.9 days. According to our derived rotational period (5.0-6.7 days), GW Ori A is far away from the

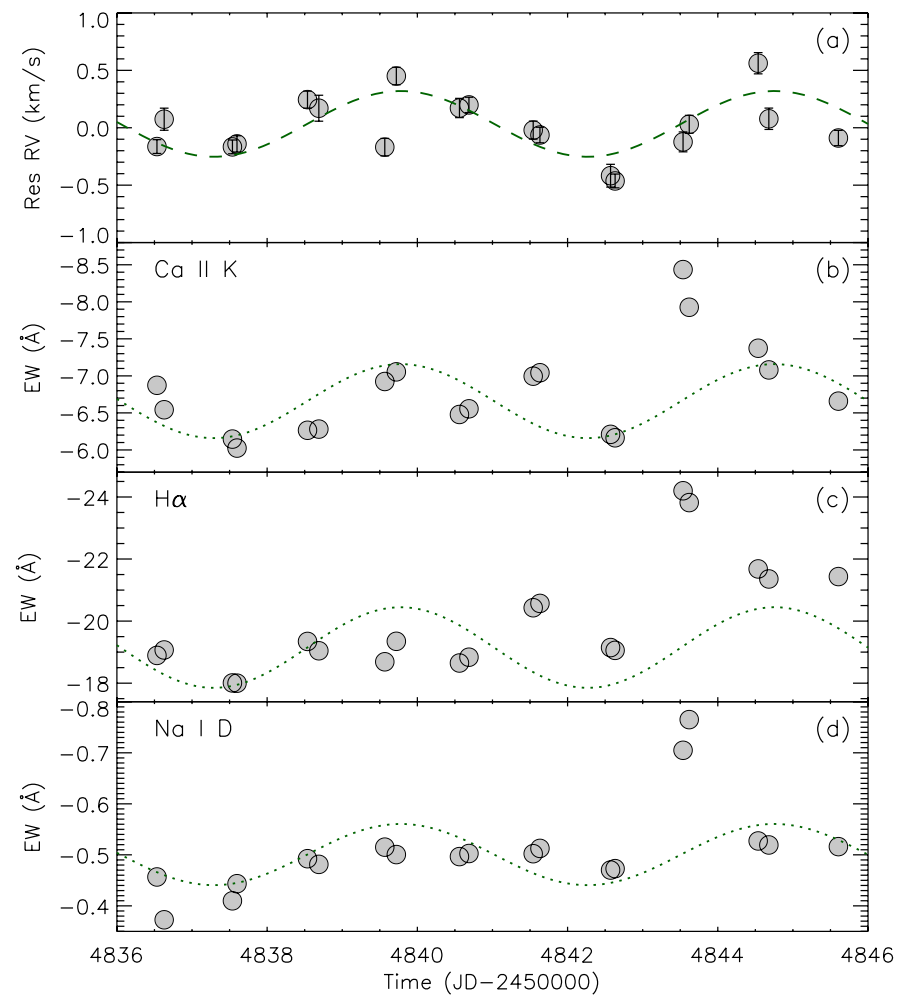

Fig. 6. Same as in Fig. 5 but for different observational dates.

limit. Given $v \sin i_{*}=43.7 \mathrm{~km} \mathrm{~s}^{-1}$, we calculate the inclination $\left(i_{*}\right)$ of the stellar rotation axis of GW Ori A, which is around $35-50^{\circ}$. Bouvier (1990) derived a shorter rotation period ( 3.3 days) for GW Ori A mainly based on the $U$-band photometry. It is unknown which period is more accurate, since both our data and theirs are not sampled very well over the time. If the rotational period of GW Ori A was the shorter one, the inclination would be around $22^{\circ}$. Here, we consider that the inclination of the rotational axis is between $22-50^{\circ}$, although current data cannot give any constraints on the inclination of the orbital axis of GW Ori A/B. If the orbital axis of GW Ori A/B is aligned with the rotational axis of GW Ori A, the mass of GW Ori B is $0.3-0.7 M_{\odot}$. However, an intermediate inclination of the orbital axis of GW Ori A/B contradicts the observed eclipses by Shevchenko et al. (1998). The eclipses were detected during 1987-1992 and then disappeared (Shevchenko et al. 1998). Therefore, more data are required to understand the nature of the eclipses and, furthermore, to give a constraint on the inclination of the the orbital axis of GW Ori A/B.

\subsection{Emission lines}

\subsection{1. $\mathrm{H} \alpha$ and $\mathrm{H} \beta$ emission lines}

\section{Equivalent widths and line profiles}

The $\mathrm{H} \alpha$ and $\mathrm{H} \beta$ lines are prominent in our spectra of GW Ori due to their strong and broad line profiles. In Fig. 8, we show the example residual profiles of $\mathrm{H} \alpha$ and $\mathrm{H} \beta$ lines observed during Jan. 5-14, 2009. The residual line profiles are obtained by normalizing the observed spectra and subtracting the photospheric absorption features from the normalized synthetical spectrum with $T_{\text {eff }}=5500 \mathrm{~K}$. As shown in Fig 8, the $\mathrm{H} \alpha$ and $\mathrm{H} \beta$ lines show very similar profiles. In both lines, the most notable feature 


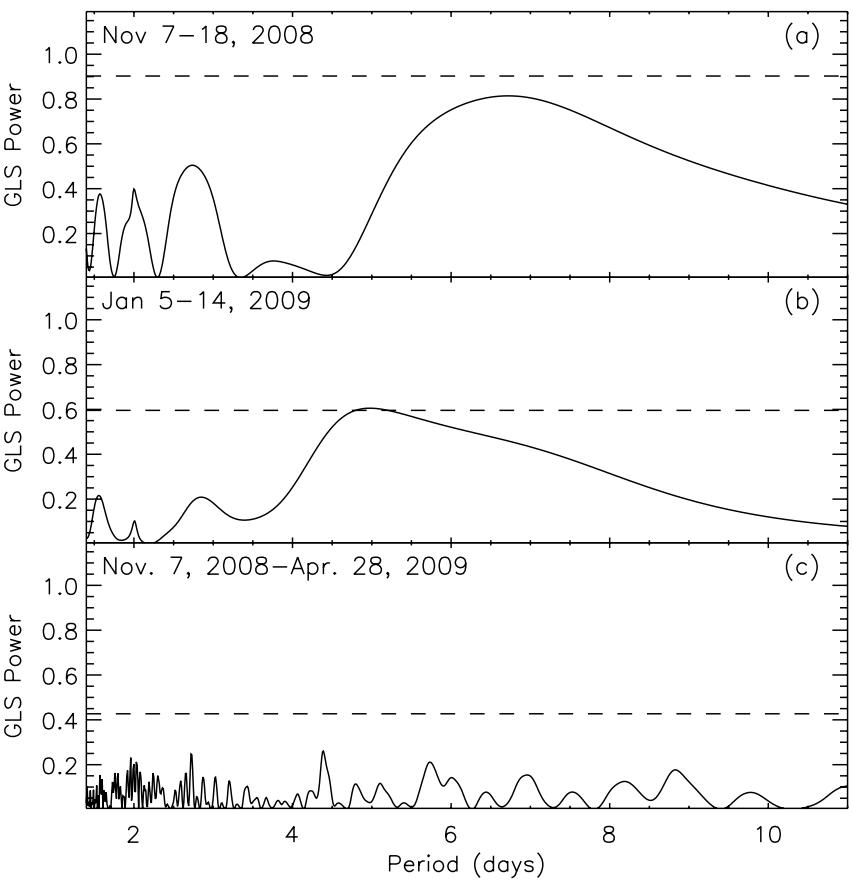

Fig. 7. a) Generalized Lomb-Scargle periodogram of the RV residuals of GW Ori during Nov. 7-11, 2008. b) The same periodogram for the RV residuals but for data obtained during Jan. 5-14, 2009. c) Same as in panels a), b) but for data taken between Nov. 7, 2008 and Apr. 28, 2009. The dashed line in each panel indicates the power level for an FAP of 0.01 , as computed by GLS.

is a blue-shifted absorption, which changes in both strength and central velocity with time. In Table 4, we list the EWs of $\mathrm{H} \alpha$ and $\mathrm{H} \beta$ calculated with the residual line profiles.

\section{Line variance}

In Figs. 9 and 10, we show the average residual profiles of $\mathrm{H} \alpha$ and $\mathrm{H} \beta$ lines with the normalized variance profiles, as calculated with the data observed during 2009 Jan. 1-14, and all the observations. The variance profiles, as defined in Johns \& Basri (1995a), are measurements of variability in each velocity bin within the lines. The variance profiles of $\mathrm{H} \alpha$ and $\mathrm{H} \beta$ show blue-shifted peaks, corresponding to the variable absorption features (see Fig. 8), and are featureless on the red side.

\section{Correlation matrices}

We calculate autocorrelation matrices for the $\mathrm{H} \alpha$ and $\mathrm{H} \beta$ lines and use them to investigate how the variations of line profiles are correlated across the lines (see Johns \& Basri 1995a). The resulting matrices are illustrated in Fig. 11. For $\mathrm{H} \alpha$, the autocorrelation matrix shows a clear correlation between the blue $\left(-300-0 \mathrm{~km} \mathrm{~s}^{-1}\right)$ and the red $\left(0-200 \mathrm{~km} \mathrm{~s}^{-1}\right)$ sides of profiles. The autocorrelation matrix of $\mathrm{H} \beta$ exhibits a similar pattern to that of $\mathrm{H} \alpha$ but with less significance. In Fig. 12, we display the linear correlation coefficient between the $\mathrm{H} \alpha$ and $\mathrm{H} \beta$ profiles within each velocity bin. The $\mathrm{H} \alpha$ and $\mathrm{H} \beta$ variations are correlated well across the whole line profiles $\left(-400-400 \mathrm{~km} \mathrm{~s}^{-1}\right)$.

\section{Decomposition of the $\mathrm{H} \alpha$ and $\mathrm{H} \beta$ line profiles}

We decompose the $\mathrm{H} \alpha$ and $\mathrm{H} \beta$ line profiles of GW Ori using multi-Gaussian functions, as done in Sicilia-Aguilar et al. (2012). We find that all the $\mathrm{H} \alpha$ and $\mathrm{H} \beta$ line profiles can be fittted well with two emission components, of which one is strong and broad and the other is narrow and weak, and one blue-shifted absorption component. Figure 13 shows the examples of fits to the $\mathrm{H} \alpha$ and $\mathrm{H} \beta$ line profiles. For $\mathrm{H} \alpha$ line, the two emission components always peak at $\sim 0 \mathrm{~km} \mathrm{~s}^{-1}$ with respect to the rest frame of GW Ori and show mean EWs of $-21.6 \pm 2.9$ and $-3.5 \pm 0.6 \AA$, respectively, for the broad and narrow components. The mean full widths of $\mathrm{H} \alpha$ at $10 \%\left(\mathrm{FW}_{\mathrm{H} \alpha, 10 \%}\right)$ of the peak intensity are $509 \pm 13$ and $156 \pm 16 \mathrm{~km} \mathrm{~s}^{-1}$ for the two components. There are no correlations between the two emission components in both the EWs and line widths, indicating they may originate from different physical processes. According to the criteria for distinguishing accretors and non-accretors using $\mathrm{FW}_{\mathrm{H} \alpha, 10 \%}$ and $\mathrm{EW}$ of $\mathrm{H} \alpha$ in Fang et al. (2009, 2013a), the narrow $\mathrm{H} \alpha$ emission component could be mainly related to the chromospheric activity, while the broad one should be due to the accretion activity.

The two emission components from $\mathrm{H} \beta$ decomposition are similar to those from $\mathrm{H} \alpha$ and peak around $\sim 0 \mathrm{~km} \mathrm{~s}^{-1}$ with respect to the rest frame of GW Ori. For the broad component, the mean full width of $\mathrm{H} \beta$ at $10 \%\left(\mathrm{FW}_{\mathrm{H} \beta, 10 \%}\right)$ of the peak intensity is $541 \pm 25 \mathrm{~km} \mathrm{~s}^{-1}$, and the mean EW is $-3.4 \pm 0.6 \AA$. The two values are $128 \pm 17 \mathrm{~km} \mathrm{~s}^{-1}$ and $-0.4 \pm 0.1 \AA$, respectively, for the narrow emission component. We note that there are correlations between the $\mathrm{H} \alpha$ and $\mathrm{H} \beta$ lines in both emission components. To test the significance of the correlation between them, we apply a Kendall $\tau$ test. If two datasets are fully correlated, the test returns a value of $\tau=1$. If they are anti-correlated, we get $\tau=-1$, and if they are independent, $\tau$ has a value of 0 . The Kendall $\tau$ test also returns a probability $p$, which is smaller when the correlation is more significant. For the broad components, the Kendall $\tau$ test yields $\tau=0.45$ and $p=5 \times 10^{-7}$ for $\mathrm{FW}_{\mathrm{H} \alpha, 10 \%}$ and $\mathrm{FW}_{\mathrm{H} \beta, 10 \%}$, and $\tau=0.62$ and $p=0$ for the EWs of both lines. The correlations for the narrow components of the $\mathrm{H} \alpha$ and $\mathrm{H} \beta$ lines are less significant with $\tau=0.30$ and $p=1 \times 10^{-3}$ from the Kendall $\tau$ test for $\mathrm{FW}_{\mathrm{H} \alpha, 10 \%}$ and $\mathrm{FW}_{\mathrm{H} \beta, 10 \%}$ and $\tau=0.38$ and $p=3 \times 10^{-5}$ for the EWs of both lines.

The blue-shifted absorption components in the $\mathrm{H} \alpha$ and $\mathrm{H} \beta$ line profiles can be related to the wind activity of GW Ori. The absorption components are variable in both the central velocities and the line strengths for both $\mathrm{H} \alpha$ and $\mathrm{H} \beta$ lines, which induces the peaks on the variance at the blue side of their line profile (see Figs. 9 and 10). We note that the absorption components in the two lines are strongly correlated in both the central velocities and the EWs. The Kendall $\tau$ test yields $\tau=0.53$ and $p=0$ for their central velocities and $\tau=0.57$ and $p=0$ for their EWs. In Fig. 14, we show the central velocities and EWs for the absorption components in $\mathrm{H} \alpha$ line profiles with respect to the orbital phases of GW Ori B. We note the variations of the two values seem to be periodic with a period comparable to orbital period of GW Ori B. This periodic variation of the central velocity and the line strengths is more notable from the mean values within individual phase bins, which are also shown in Fig. 14. The median central velocity of the wind component is around $-70 \mathrm{~km} \mathrm{~s}^{-1}$, which is much smaller than the escape velocity $\left(\sim 440 \mathrm{~km} \mathrm{~s}^{-1}\right)$ at the surface of GW Ori A and comparable to the escape velocity from the disk at a distance of 1.4 AU. Thus, the blue-shifted absorption component should be attributed to disk winds launched near the orbit of GW Ori B and could be affected by the orbital motion of GW Ori B. Furthermore, we also note that the EWs of blue-shifted absorption components in Fig. 14 are usually larger when they are bluer, which can be due to that wind speeds and EWs are usually correlated in powerful winds. The blue-shifted absorption components in $\mathrm{H} \beta$ line profiles show similar variations with the orbital phases of GW Ori B to those of $\mathrm{H} \alpha$ lines. 


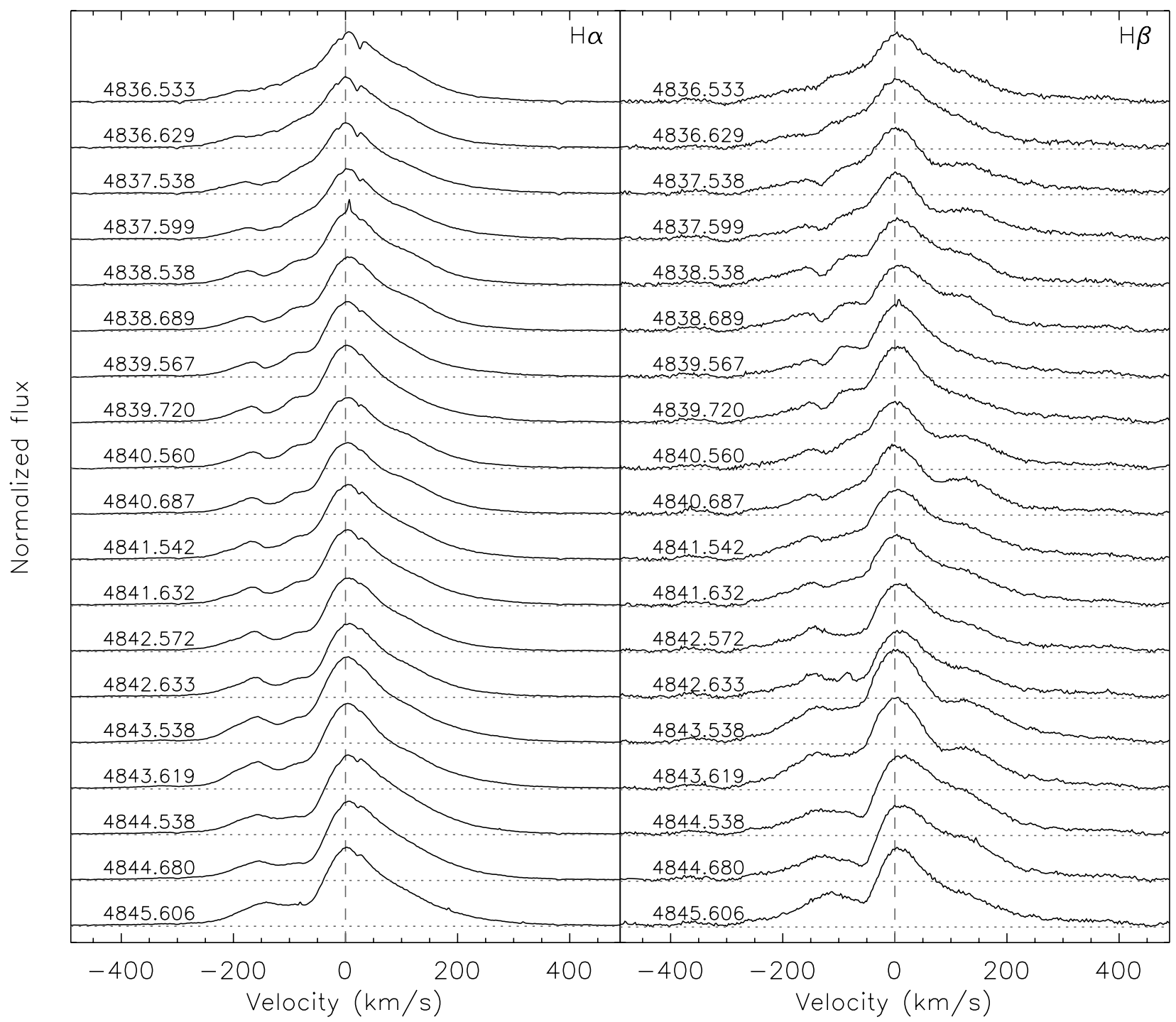

Fig. 8. Residual line profiles of $\mathrm{H} \alpha$ and $\mathrm{H} \beta$ observed during Jan. 5-14. The profiles have been shifted for clarity. The vertical dashed lines mark the spectral line center at the stellar rest frame, and the horizontal dotted lines show the continuum level. The numbers on the left are the Julian dates of observation minus 2450000 .

\subsubsection{Other emission lines}

Besides $\mathrm{H} \alpha$ and $\mathrm{H} \beta$, we also detect other emission lines in the spectra of GW Ori, including the $\mathrm{H} \gamma$ Balmer line, Ca II lines at $3933,3968,8498$, and $8662 \AA^{4}$, He I $\lambda 5876$, O I lines at 6300 , 6363,7773 , and $8446 \AA$, and NaI D lines at 5890/5896 $\AA$. In Fig. 15 we show the residual profiles of these emission lines obtained on Nov. 8, 2007 as an example. All lines except the oxygen doublet at 6300 and $6363 \AA$ exhibit broad profiles. In Table 4, we list the EWs of these lines calculated from their residual line profiles (see the definition of residual line profiles in Sect. 3.3.1a).

The $\mathrm{H} \gamma$ line in GW Ori is much weaker than $\mathrm{H} \alpha$ and $\mathrm{H} \beta$ and appears in emission only after the subtraction of the photospheric absorption. However, the residual profiles of the $\mathrm{H} \gamma$ lines are very similar to those of the $\mathrm{H} \alpha$ and $\mathrm{H} \beta$ lines. In Fig. 15, the He I $\lambda 5876$ line clearly shows double peaks centered at $\sim-100 \mathrm{~km} \mathrm{~s}^{-1}$ and $\sim 100 \mathrm{~km} \mathrm{~s}^{-1}$. This kind of He I $\lambda 5876$ line profile is quite atypical among T Tauri stars

\footnotetext{
4 The Ca II line at $8542 \AA$ falls at the edge of the FEROS gap and is thus excluded it here.
}

(see e.g., Muzerolle et al. 1998; Beristain et al. 2001). In Fig. 15, the CaII lines at $3933,3968,8498$, and $8662 \AA$ and the Na I D lines at $5890 / 5896 \AA$ show broad line profiles with blueward asymmetry. All these above described lines have been widely used as tracers of accretion in the literature (Mohanty et al. 2005; Herczeg \& Hillenbrand 2008; Fang et al. 2009; Rigliaco et al. 2012). The OI lines at 7773 and $8446 \AA$ are also broad and could be related to accretion activity. Two forbidden lines [OI] $\lambda 6300 / 6363$ show very narrow line profiles with slightly blue-shifted peaks. They are proposed to be mainly due to prompt emission following UV photodissociation of OH molecules (Storzer \& Hollenbach 1998). However, the atmosphere of Earth can also produce strong oxygen forbidden lines. Our observations may be contaminated by telluric emission.

\subsection{Accretion}

\subsubsection{Accretion-related emission lines}

The GW Ori system has a low eccentricity $(e=0.18)$ and, thus, is expected to show a smooth enhancement in the accretion rates 


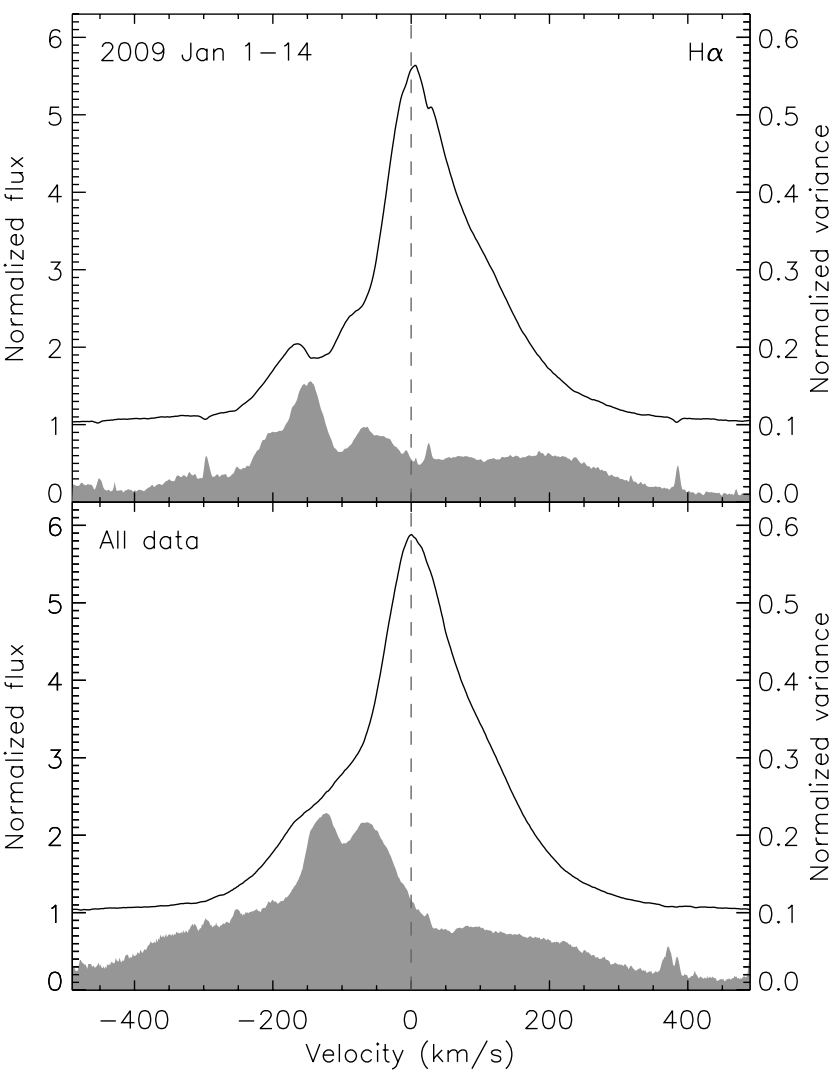

Fig. 9. Average $\mathrm{H} \alpha$ line profiles (solid line) and normalized variance profiles (gray shaded areas) calculated with residual spectra. The vertical dashed lines mark the spectral line center at the stellar rest frame.

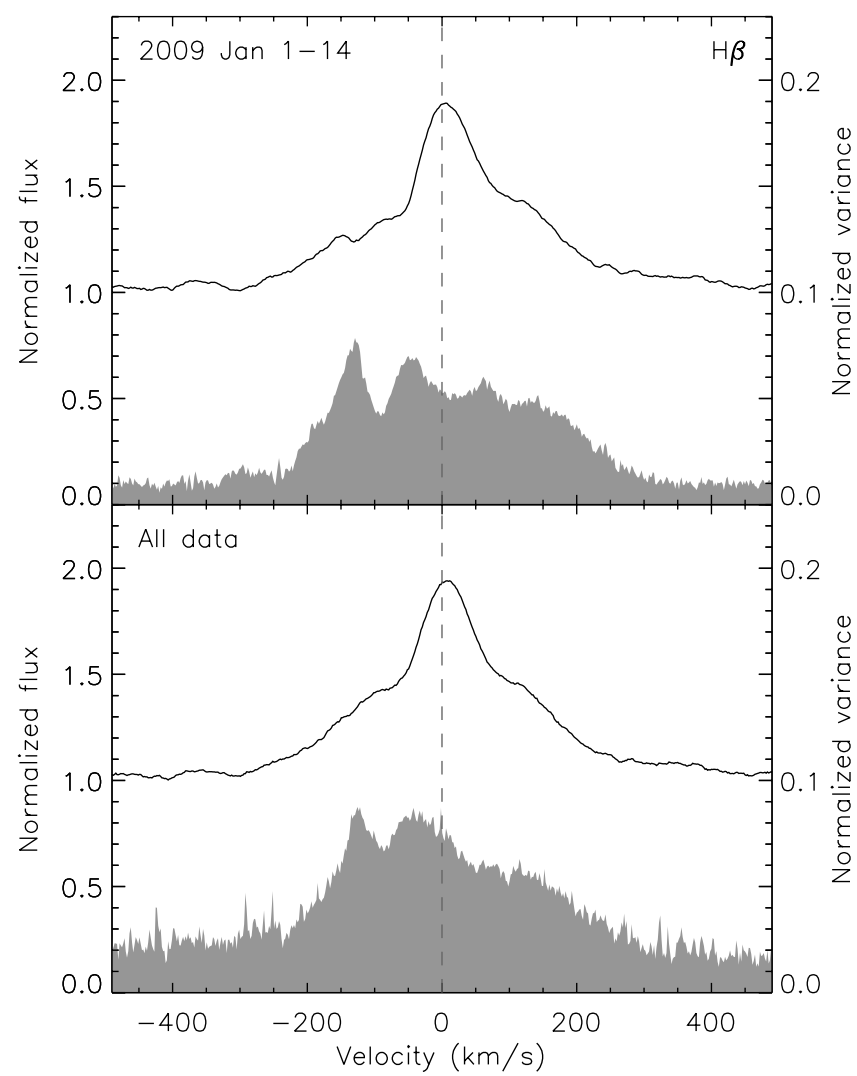

Fig. 10. Similar to Fig. 9 but for $\mathrm{H} \beta$ line.
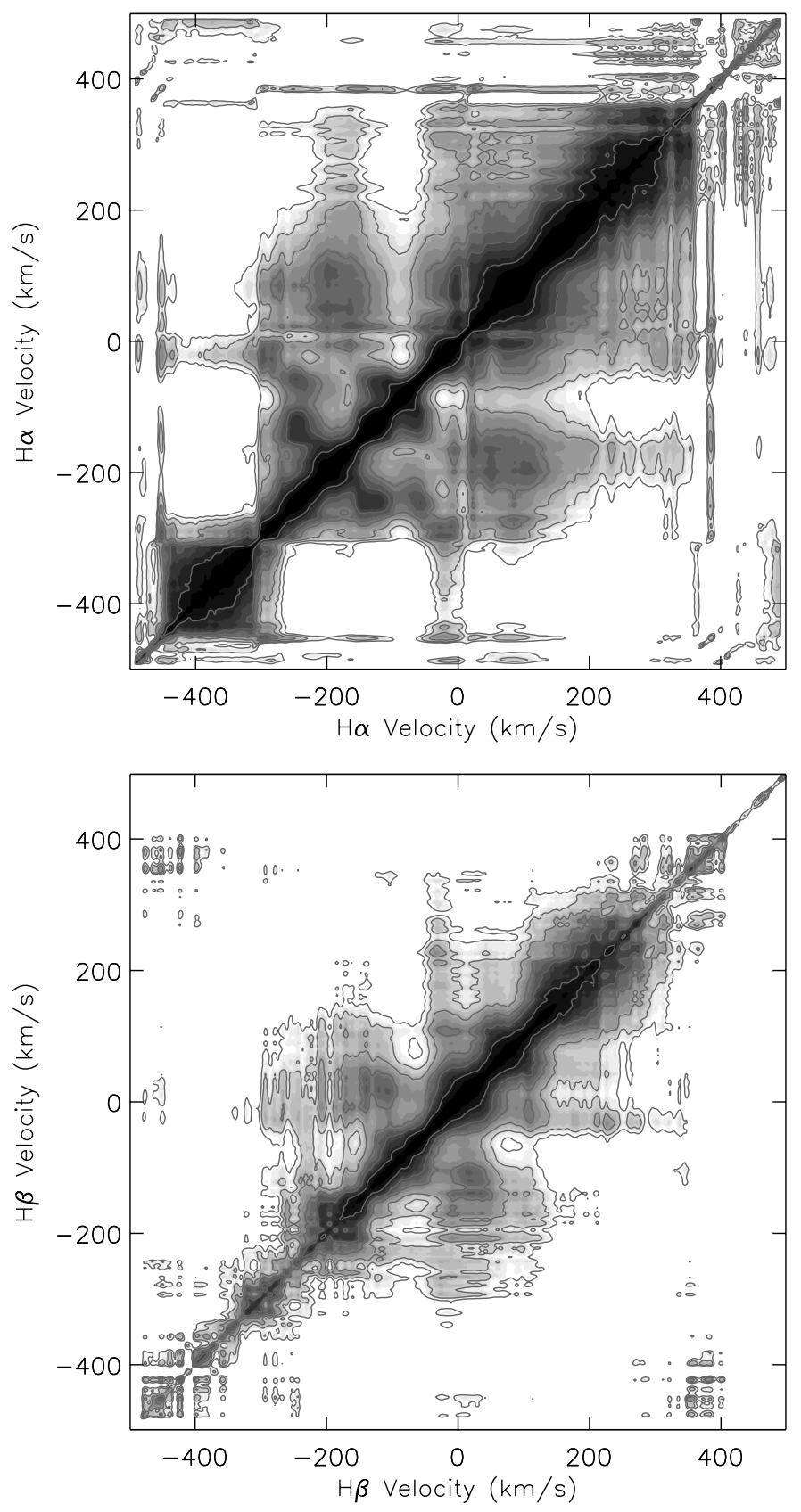

Fig. 11. Correlation matrices of $\mathrm{H} \alpha$ and $\mathrm{H} \beta$ lines for GW Ori. The lowest contours in each panel corresponds to $99.9 \%$ confidence level.

with the orbital phases (Artymowicz \& Lubow 1996). In Fig. 16, we show the $\mathrm{FW}_{\mathrm{H} \alpha, 10 \%}$ for the broad components (produced in accretion processes, see Sect. 3.3.1d) from the line decomposition, the EWs of the $\mathrm{H} \alpha$ line, and the EWs of Ca II $\lambda 3933$ line with respect to the orbital phases of GW Ori B. Both lines are good tracers of accretion. Note that our observations are not uniformly sampled over the orbital phases, which makes it hard to draw any definite conclusions. However, some hints can be seen in Fig. 16. The $\mathrm{FW}_{\mathrm{H} \alpha, 10 \%}$ values are distributed around $510 \mathrm{~km} \mathrm{~s}^{-1}$ over all the orbital phases. At some time we see that $\mathrm{FW}_{\mathrm{H} \alpha, 10 \%}$ reachs $550 \mathrm{~km} \mathrm{~s}^{-1}$ at the orbital phases around $\sim 0.4$, corresponding to an increase of accretion rates by a factor of $2-3$. The EWs of $\mathrm{H} \alpha$ and $\mathrm{Ca}$ II $\lambda 3933$ lines are scattered around -23 and $-5 \AA$, respectively over all the orbital phases. We note sometimes the EWs of both lines increase by a factor of $\sim 1.5$ at 


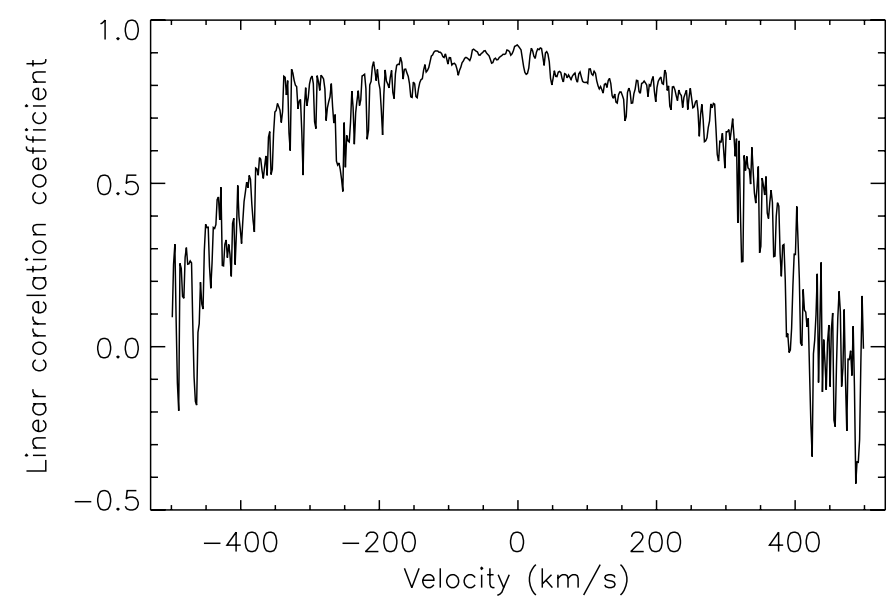

Fig. 12. Linear correlation coefficient between $\mathrm{H} \alpha$ and $\mathrm{H} \beta$ line profiles in each velocity bin.

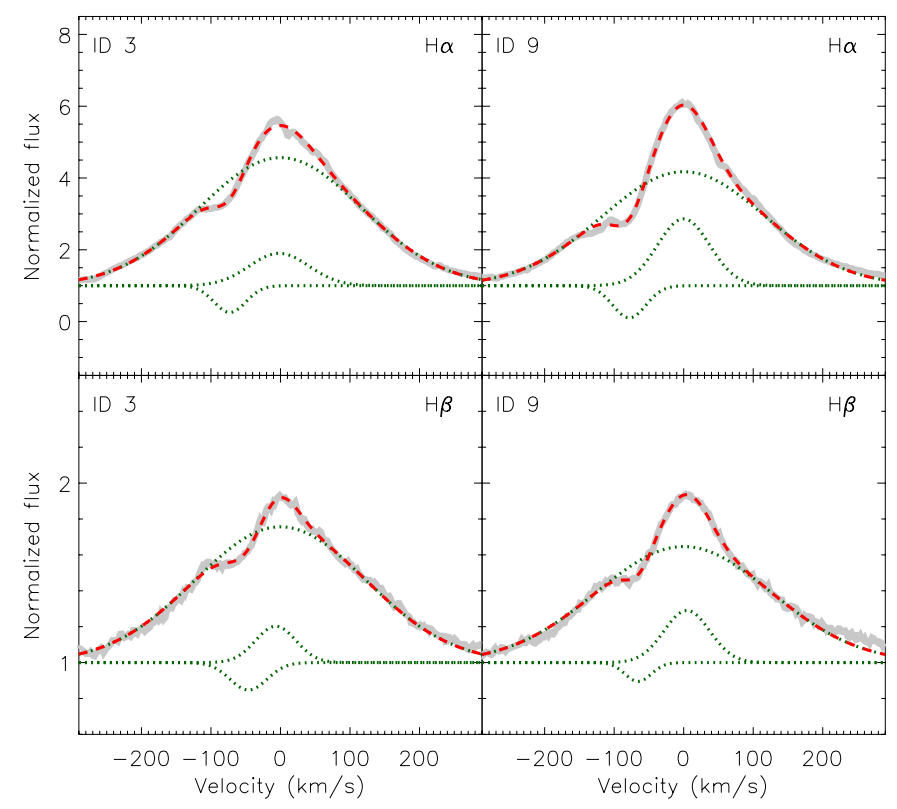

Fig. 13. Examples of decomposition of $\mathrm{H} \alpha$ and $\mathrm{H} \beta$ line profiles using three Gaussian components. In each panel, the thick gray line is the observed spectrum; the dotted lines display individual components, and the dashed line shows the final fit. The zero velocity is that of the GW Ori rest frame.

the orbital phases around $\sim 0.5$, suggesting an increase of accretion rate by a factor of $\sim 2$, according to the relations between the line luminosity of $\mathrm{H} \alpha$ and $\mathrm{Ca}$ II $\lambda 3933$ to the accretion luminosity (Fang et al. 2009; Herczeg \& Hillenbrand 2008).

We divide the $\mathrm{FW}_{\mathrm{H} \alpha, 10 \%}$ and $\mathrm{EW}$ values into different phase bins and show their mean values and the standard deviations in Fig. 16. The mean $\mathrm{FW}_{\mathrm{H} \alpha, 10 \%}$ is quite constant over all the phase bins with a slightly larger standard deviation at phase $\sim 0.5$ due to the occasional enhancement of accretion at this bin. The mean EWs of $\mathrm{H} \alpha$ line seem to change from bin to bin, but the variations are small with the lowest mean EW of $\sim-20 \AA$ at the phase bin $\sim 0.3$ and the highest mean EW of $\sim-26 \AA$ at the phase bin $\sim 0.7$. Similar to the $\mathrm{FW}_{\mathrm{H} \alpha, 10 \%}$ the standard deviation of $\mathrm{H} \alpha \mathrm{EWs}$ at the phase bin $\sim 0.5$ is larger than those of others. The mean EWs of the CaII $\lambda 3933$ line are very constant within different phase bins, although a slight increase of the mean EW is noted at the phase bin $\sim 0.5$. In Fig. 17, we show the median line profiles

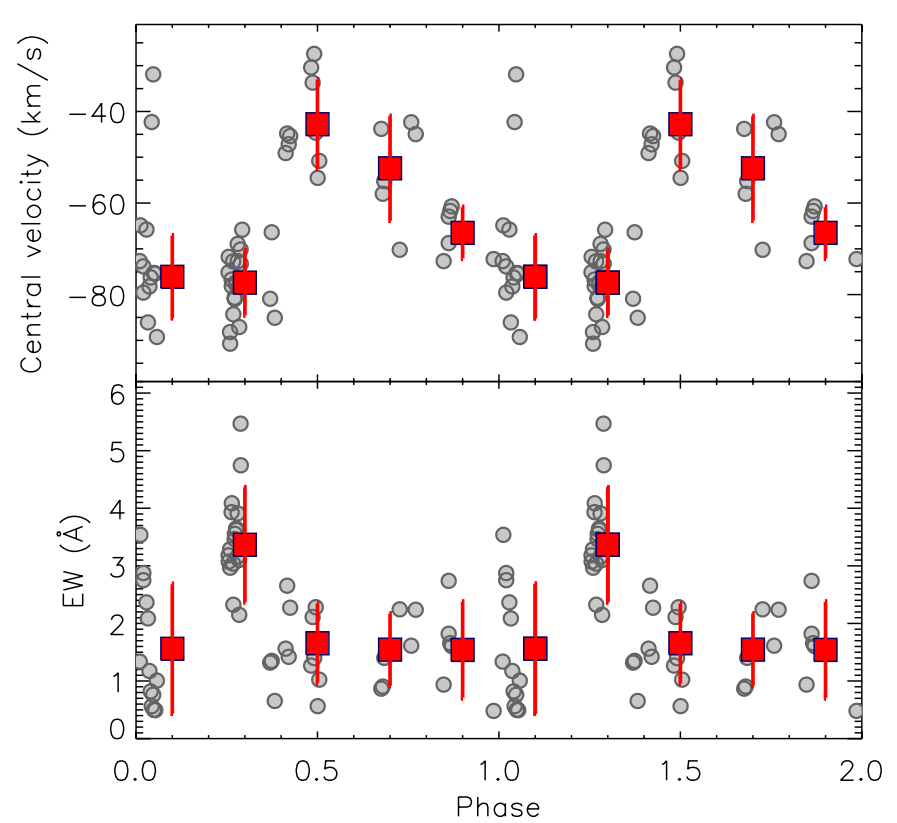

Fig. 14. a) Central velocities of the disk wind components from the decomposition of $\mathrm{H} \alpha$ line profiles, as plotted over the orbital phases of GW Ori B. The velocity is in the rest frame of GW Ori. We divide the orbital phases into different bins and show the mean value of central velocities in each bin with a filled square and its standard deviation with an error bar. b) EWs of the disk wind components, as shown in panel a), plotted over the orbital phases of GW Ori B. We divide the orbital phases into different bins and show the mean value of EWs in each bin with a filled square and its standard deviation with an error bar.

of $\mathrm{H} \alpha$ and Ca II $\lambda 3968$ within four bins of orbital phases, 0-0.25, $0.25-0.5,0.5-0.75$, and $0.75-1.0$. For both $\mathrm{H} \alpha$ and Ca II $\lambda 3968$, we see the line profiles within the phase bins of $0-0.25,0.25-$ 0.5 , and $0.75-1.0$ are quite similar, and the line profiles within the phase bin of $0.5-0.75$ are stronger than others.

We have examined other accretion-related emission lines, such as $\mathrm{H} \beta, \mathrm{H} \gamma, \mathrm{NaID}$, and other Ca II lines. These lines exhibit similar behaviors to those of $\mathrm{H} \alpha$ and Ca II $\lambda 3933$, where the EWs of these lines are scattered around constant values over all the orbital phases of GW Ori B with a probable accidental increase of accretion at the phases around 0.5 . Since our observations do not cover the phases before and after the high accretion value at the same orbital period and, thus, we do not know the accretion rates before and after phase 0.5 , it is not possible to establish whether the enhancement of accretion at the phases $\sim 0.5$ is due to the orbital modulation or to an unrelated, more extended episode of increased accretion.

Here, we use the EWs of accretion-related emission lines as proxy of accretion of GW Ori. However, the variations of the line EWs could be also attributed to variable brightness of a star. Long-term monitoring of GW Ori with imaging has revealed that GW Ori is a variable source (Grankin et al. 2007). Furthermore, the strength of some accretion-related emission lines, such as $\mathrm{H} \alpha, \mathrm{H} \beta, \mathrm{Ca}$ II lines, and etc., may be contaminated by the stellar and disk winds, or be modulated by the stellar rotation (see Figs. 5 and 6) if the accretion columns are not uniformly distributed on the stellar surface (Kurosawa \& Romanova 2013). These factors may in part produce the scatter seen in Fig. 16. 

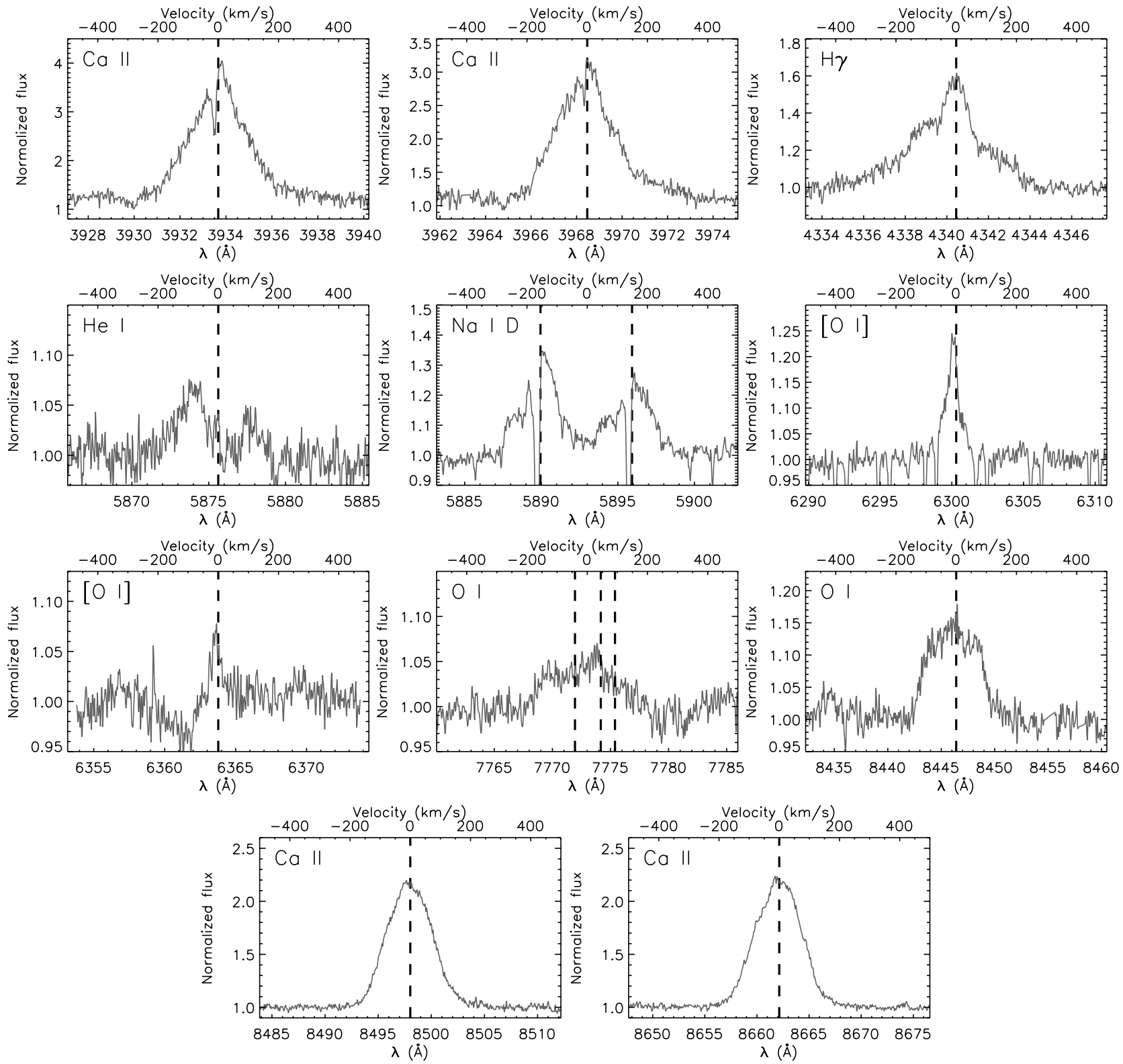

Fig. 15. Example profiles of the emission lines detected in the optical spectrum (ID 1 in Table 1) of GW Ori. The vertical dashed lines mark the spectral line center at the stellar rest frame. For the O I triplet at $7773 \AA$, the central wavelength for each line is marked. The central absorption components in Ca II at 3933 and $3968 \AA$ and in Na I at 5890/5896 $\AA$ are due to the absorption from interstellar medium.

\subsubsection{U-band excess}

The $U$-band excess is an excellent proxy for accretion rates and has been wide used in the literature (Gullbring et al. 1998; Hartmann et al. 1998; Sicilia-Aguilar et al. 2010; Mendigutía et al. 2011; Rigliaco et al. 2011). The object GW Ori has been simultaneously observed for 20 years (1983-2003) in $U$ and other optical bands (Bouvier et al. 1988; Grankin et al. 2007). With these data, which have not been used to study accretion properties of GW Ori in the literature, we can properly calculate the accretion rates from $U$-band excess and study variability of accretion at a timescale of several ten years. We use the observed $V-R$ color to estimate the visual extinction. For the photometric data in the Johnson system from Grankin et al. (2007), we first convert them to Cousins system by using the relation from Landolt (1983): $(V-R)_{\mathrm{C}}=-0.0320+0.71652 \times(V-R)_{J}$.
The visual extinction is then estimated by comparing the observed $(V-R)_{\mathrm{C}}$ color with the intrinsic $(V-R)_{\mathrm{C}}$ color for synthetical spectra with $T_{\text {eff }}=5500 \mathrm{~K}$ and $\log g=3.5$ in Pecaut \& Mamajek (2013) and by using an extinction law in Landolt $U V R$ bands from Schlafly \& Finkbeiner (2011) in the case of $R_{V}=3.1$. We use the visual extinction to deredden the photometry. The $U$-band magnitude of the stellar photosphere is obtained from the dereddened V-band photometry and an intrinsic $U-V$ color for synthetical spectra with $T_{\text {eff }}=5500 \mathrm{~K}$ and $\log g=3.5$ in Pecaut \& Mamajek (2013). We convert the $U$-band photometry to $U$-band luminosity using the zero-point flux and bandwidth for $U$ band $\left(4.19 \times 10^{-9} \mathrm{erg} \mathrm{s}^{-1} \mathrm{~cm}^{-2} \AA^{-1}\right.$ and $680 \AA$, respectively; Sicilia-Aguilar et al. 2010). We derive the $U$-band excess emission by subtracting the photospheric emission from the dereddened emission of GW Ori in $U$ band and then convert it to accretion luminosity according to the empirical relation 


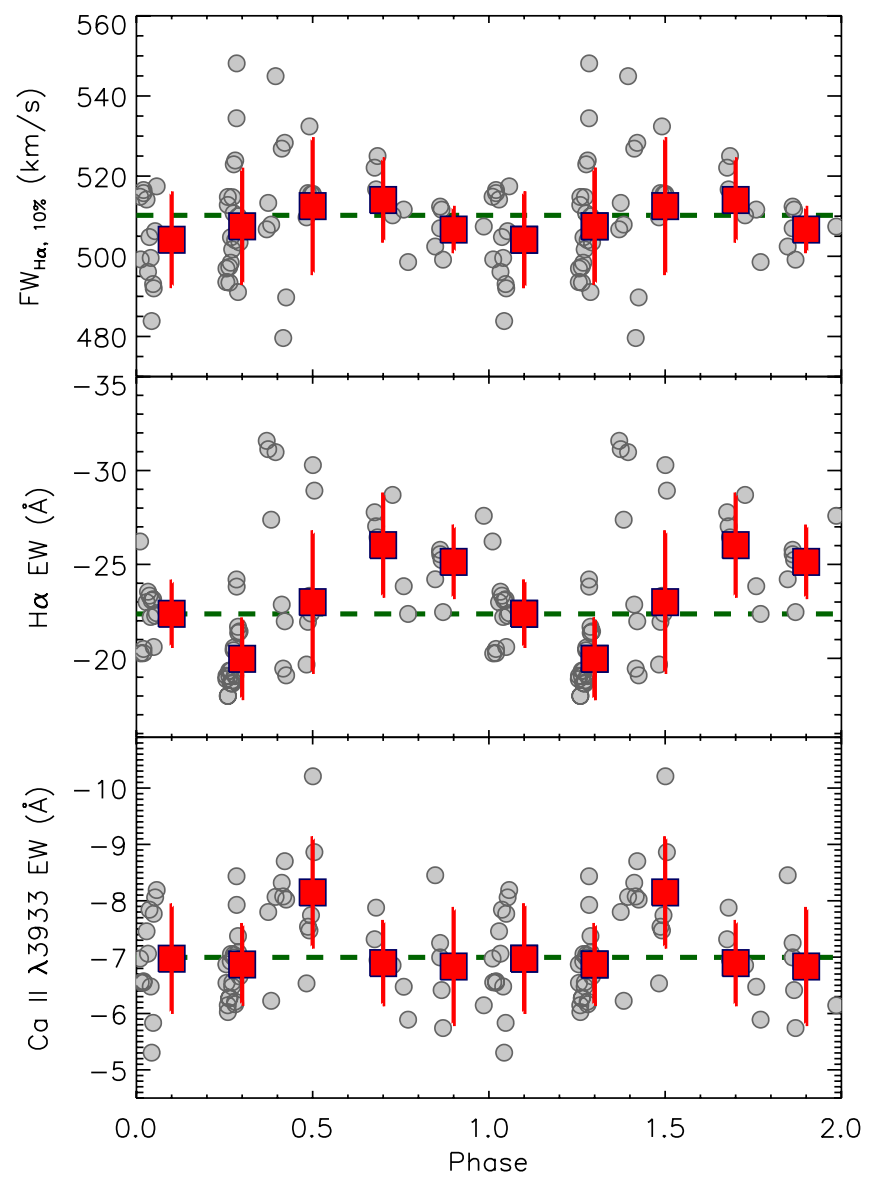

Fig. 16. $\mathrm{FW}_{\mathrm{H} \alpha, 10 \%}$ values for the broad components from the $\mathrm{H} \alpha$ line decomposition, and EWs of $\mathrm{H} \alpha$ and Ca II $\lambda 3933$ with respect to the orbital phases of GW Ori B. We divide the orbital phases into different bins and show the mean values in individual bins with filled squares, as well their standard deviations with error bars. The dashed line in each panel is the median value.

in Gullbring et al. (1998). The inferred accretion luminosities are then converted into mass accretion rates using the following relation:

$\dot{M}_{\mathrm{acc}}=\frac{L_{\mathrm{acc}} R_{\star}}{G M_{\star}\left(1-\frac{R_{\star}}{R_{\mathrm{in}}}\right)}$,

where $R_{\text {in }}$ denotes the truncation radius of the disk, which is taken to be $5 R_{\star}$ (Gullbring et al. 1998). The parameter $G$ is the gravitational constant, $M_{\star}$ and $R_{\star}$ is the stellar mass and radius of GW Ori A, respectively.

We find that GW Ori shows excess emission in all collected $U$-band photometry, suggesting that GW Ori has been accreting for 20 years. In total, we have 307 estimates of the accretion rate for GW Ori during the period of 1983-2003. The typical uncertainty of measurement is $\sim 2 \times 10^{-7} M_{\odot} \mathrm{yr}^{-1}$. The mean accretion rate of GW Ori is $\sim 4 \times 10^{-7} M_{\odot} \mathrm{yr}^{-1}$, which is consistent with the result $\left(2.56-3.15 \pm 1.99 \times 10^{-7} M_{\odot} \mathrm{yr}^{-1}\right)$ in Calvet et al. (2004). In Fig. 18, we show the accretion rates of GW Ori with respect to the orbital phases of GW Ori B. Similar to Fig. 16, our data are not uniformly sampled over the orbital phases. Therefore, we would not make any conclusion from it. Instead, we only describe the hints from Fig. 16. We note that the accretion rates of GW Ori are scattered around $\sim 4 \times 10^{-7} M_{\odot} \mathrm{yr}^{-1}$ over all orbital phases, indicating that the
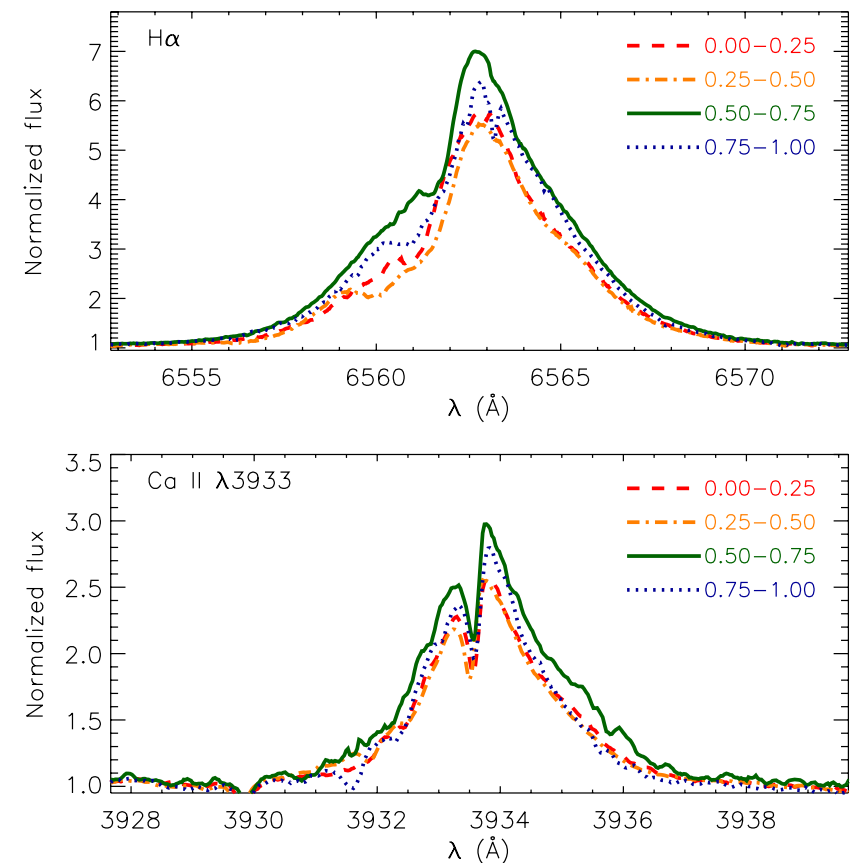

Fig. 17. Median line profiles of $\mathrm{H} \alpha$ and Ca II $\lambda 3933$. The line profiles are within four phase bins, $0-0.25,0.25-0.5,0.5-0.75$, and $0.75-1.0$, and are shown with the dashed lines, dash-dotted lines, solid lines, and dotted lines, respectively.

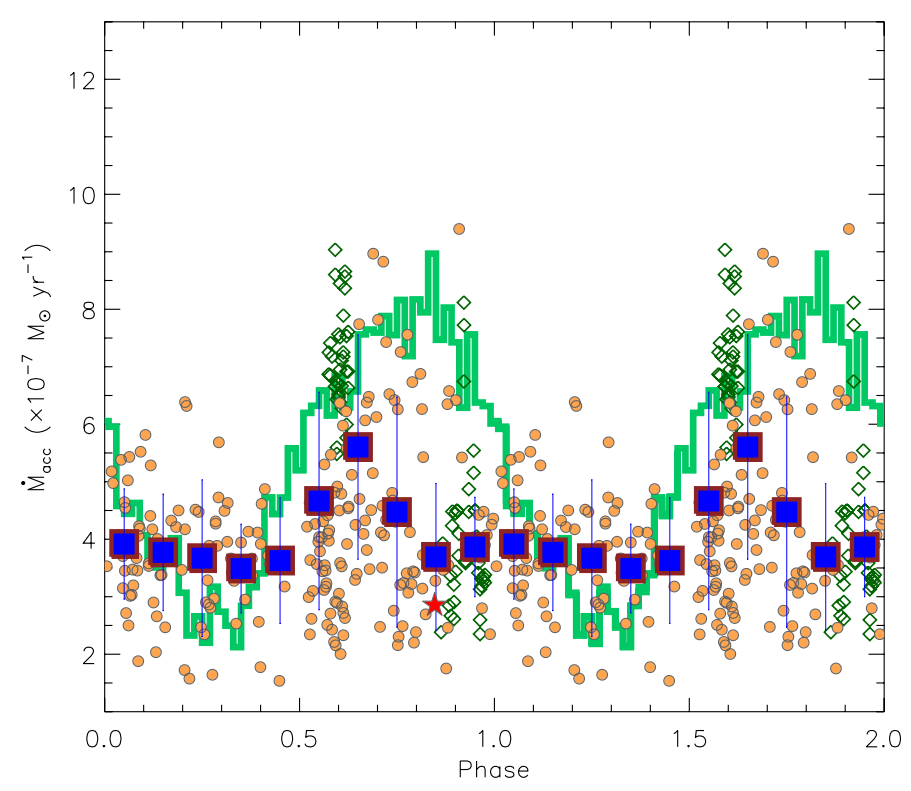

Fig. 18. Accretion rates derived from $U$-band excesses of GW Ori, as plotted over the orbital phases of GW Ori B. The filled circles show the accretion rates estimated from the data in Grankin et al. (2007); the open diamonds are for the accretion rates derived from the data in Bouvier et al. (1988), and the star symbol mark the accretion rate from Calvet et al. (2004). We divide the orbital phases into different bins and show the mean value of accretion rates in each bin with a filled square, as well as its standard deviation with an error bar. The solid line show theoretical prediction for the total accretion rate of a close binary system in the low-eccentricity case from Artymowicz \& Lubow (1996). The theoretical result have been arbitrarily scaled to compare with the observations.

accretion rate of GW Ori is mostly constant. We also see that the accretion of GW Ori are occasionally enhanced by a factor 
of $\sim 2-3$ within the orbital phases of $\sim 0.6-0.8$. We divide the data into different phase bins and calculate the mean values and the standard deviations of the measurements. The results are also shown in Fig. 18. The mean values are generally constant within different phase bins. In addition, besides the phase bins around $0.6-0.8$, the standard deviations of accretion rates are $\sim 1 \times 10^{-7} M_{\odot} \mathrm{yr}^{-1}$. Within the orbital phases of $0.6-0.8$, the standard deviations are about twice the typical value within other phase bins, which is attributed to the enhancement of accretion at these phases. Similar to the accretion-related emission lines in Sect. 3.4.1, the $U$-band data also do not cover the phases before and after the enhancement of accretion at the same orbital period. Therefore, there is no evidence that the increase of the accretion rates at phases $0.6-0.8$ could be due to orbital modulation.

\subsection{Spectral energy distribution}

We construct the SED of GW Ori by using the $U B V R_{\mathrm{C}} I_{\mathrm{C}}$ photometry from Calvet et al. (2004), the $J H K_{\mathrm{s}}$ photometry from the 2MASS survey (Skrutskie et al. 2006), the photometry at $3.4,4.6,12$, and $22 \mu \mathrm{m}$ from the WISE survey (Wright et al. 2010), the photometry at 9 and $18 \mu \mathrm{m}$ from the AKARI survey (Ishihara et al. 2010), and the fluxes at 350, 450, 800, 850, 1100, $1360 \mu \mathrm{m}$ from Mathieu et al. (1995). We obtain the 5-37 $\mu \mathrm{m}$ low-resolution IRS spectrum ${ }^{5}$ and the MIPS $70 \mu$ m photometry $^{6}$ of GW Ori from the Spitzer data archive (Program ID 40145 and 20339, respectively). We do not use the fluxes from the IRAS survey. A comparison of the fluxes at IRAS $60 \mu \mathrm{m}$ and MIPS $70 \mu \mathrm{m}$ show the IRAS $60 \mu \mathrm{m}$ flux is $\sim 1.7$ times of the flux at the MIPS $70 \mu \mathrm{m}$, suggesting that the IRAS observations with poor spatial resolutions are contaminated by the dust emission in the field near GW Ori. Figure 19 shows the SED of GW Ori. The broad dip around the silicate $10 \mu \mathrm{m}$, as noted by Mathieu et al. (1991), is clearly seen in the IRS spectrum.

\subsubsection{Modeling the SED of GW Ori}

We use the 2D RADMC code from Dullemond \& Dominik (2004) to model the SED of GW Ori. The stellar parameters adopted in the models are $T_{\mathrm{eff}}=5500 \mathrm{~K}, R_{\star}=7.6 R_{\odot}$, and $M_{\star}=3.9 M_{\odot}$. In the calculations, the inner disk radius $\left(R_{\mathrm{in}}\right)$ is left as a free parameter with the disk mass $\left(M_{\text {disk }}\right)$. The outer disk radius $\left(R_{\text {out }}\right)$ is fixed to be $\sim 500 \mathrm{AU}$ (Mathieu et al. 1995). We assume a pressure scale height $\left(H_{\mathrm{P}}\right)$ that varies as a power law with the disk radius $(\mathrm{R}), H_{\mathrm{P}} / R=R^{1 / 7}$. The scale height at the outer disk radius is parameterized as $H_{\text {out }} / R_{\text {out }}$ and set to be a free parameter. The disk surface density $(\Sigma)$ is estimated from $M_{\text {disk }}$, assuming a distribution $\Sigma \propto R^{\alpha}$ and setting $\alpha=-1$. We take a gas-to-dust ratio to be 100 and use a power-law size distribution with an exponent of -3.5 for the sizes of dust with a minimum size of $0.1 \mu \mathrm{m}$ and a maximum grain size of $1000 \mu \mathrm{m}$. Two populations of amorphous dust grains (25\% carbon and $75 \%$ silicate) are included in the calculations. We assume that the disk axis is aligned with the stellar rotation axis of GW Ori (see Sect. 3.2) and use a moderate disk inclination of $\sim 40^{\circ}$ to calculate the SEDs from disk models. We vary the free parameters in disk

\footnotetext{
5 The extracted spectra are based on the droopres products processed through the S18.7.0 version of the Spitzer data pipeline. See the detail spectral extraction procedure is described in Bouwman et al. (2008).

6 The aperture photometry of GW Ori was performed with a $60^{\prime \prime}$ aperture and a sky annuli of $71.5^{\prime \prime}$ and $119.2^{\prime \prime}$, which give a flux of $19.7 \pm 0.1 \mathrm{Jy}$.
}

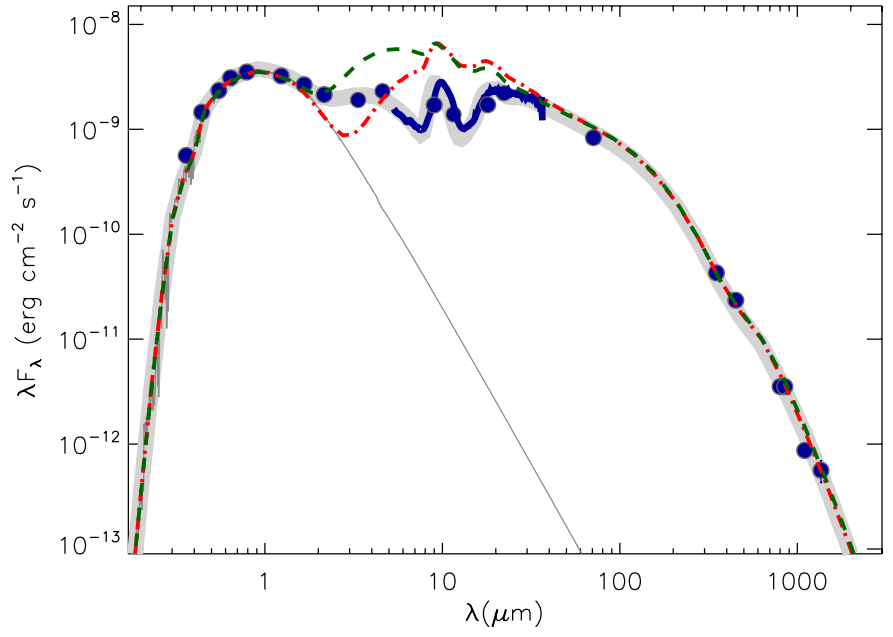

Fig. 19. Observed SED of GW Ori. The broad band photometry is shown with the filled circles, and the IRS spectrum of this source is displayed in solid line. The thick gray line shows a best-fit model. The dashed line shows the model SED for a disk with $M_{\text {disk }}=0.14 M_{\odot}$, $H_{\text {out }} / R_{\text {out }}=0.235$, and $R_{\text {in }}=2.3 \mathrm{AU}$, and the dot-dashed line display another model SED for a disk with $M_{\text {disk }}=0.14 M_{\odot}, H_{\text {out }} / R_{\text {out }}=0.2$, and $R_{\text {in }}=8 \mathrm{AU}$. The photospheric emission level is indicated with a thin gray curve.

models to calculate the SEDs. In Fig. 19, we show one example model SED calculated with $M_{\text {disk }}=0.14 M_{\odot}, H_{\text {out }} / R_{\text {out }}=0.235$, and $R_{\text {in }}=2.3 \mathrm{AU}$. The model SED has been reddened with $A_{V} \sim 1.4$ to fit the photometry of GW Ori in optical bands ${ }^{7}$. We note that the model SED can fit the observed SED at wavelengths $\gtrsim 30 \mu \mathrm{m}$ very well but exhibits much stronger excess emission at infrared wavelengths of $2-30 \mu \mathrm{m}$ than the observations, indicating that the $R_{\text {in }}$ of the disk model is too small. We increase $R_{\text {in }}$ to $8 \mathrm{AU}$ and set $H_{\text {out }} / R_{\text {out }}=0.2$ and $M_{\text {disk }}=0.14 M_{\odot}$. The calculated SED is shown in Fig. 19. Similar to the previous model, the new SED can fit the observed SED at wavelengths $\gtrsim 30 \mu \mathrm{m}$ but shows much stronger excess emissions at a wavelength range of 6-30 $\mu \mathrm{m}$ compared with the observations. We also note that the new disk model exhibits no excess emission at wavelengths $\lessgtr 3 \mu \mathrm{m}$, which is inconsistent with the observations. The above two experiments give a hint that it is hard to reproduce the SED of GW Ori using typical disk models.

Since GW Ori is a triple system with a second companion at a projected distance $\sim 8 \mathrm{AU}$, we would expect a gap in the disk created by the companions, as shown in the simulations (Artymowicz \& Lubow 1994, 1996; Pichardo et al. 2008). Such disk models with gaps can have moderate excess emission at near- and mid-infrared wavelengths by heating the small amount of dust particles in the gap. Furthermore, the strong and narrow silicate emission feature at $10 \mu \mathrm{m}$ shown on the IRS spectrum of GW Ori indicates that the dust in the gap is dominated by a small amount of tiny dust particles. To include a gap in the disk models, we add a new parameter $\left(R_{\text {gap }}\right)$ as the gap size. In the gap, the dust also consists of the two populations of amorphous dust grains with the same power-law distribution of sizes as in the outer disk but with a minimum size of $0.005 \mu \mathrm{m}$ and a maximum size of $1 \mu \mathrm{m}$. In the calculations, the model grid is refined at the edge of the gap to improve resolution in a place where opacity changes.

7 Hereafter, all the calculated SEDs are reddened to fit the SEDs of GW Ori in optical bands before we compare them with the observations. 


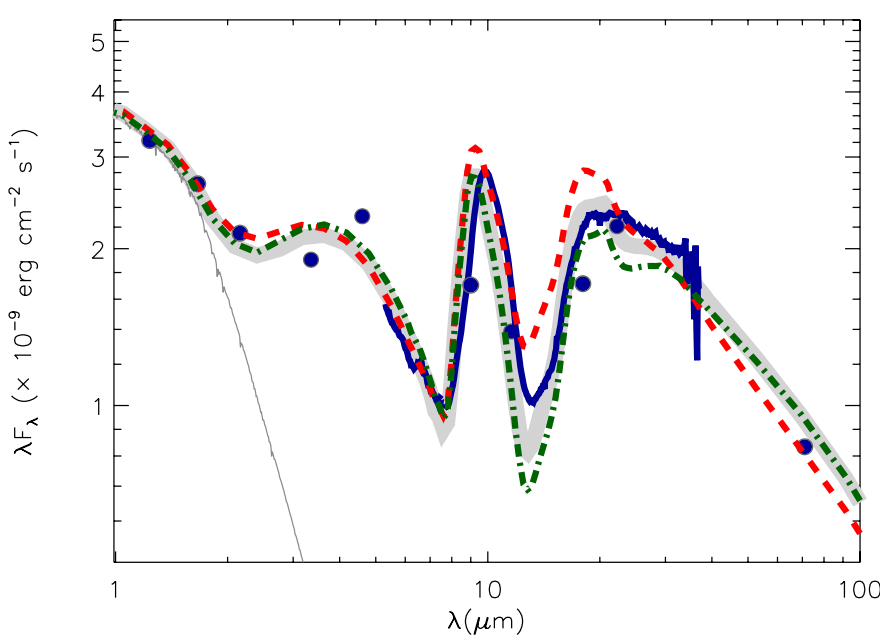

Fig. 20. Observed SED of GW Ori within a wavelength range of $1-100 \mu \mathrm{m}$. The dashed line shows the model SED for a disk with $R_{\text {gap }}=25 \mathrm{AU}$ and a dash-dotted line display another model SED for a disk with $R_{\text {gap }}=55$ AU. Others in the figures are same as in Fig. 19.

Table 5. Disk model parameters for GW Ori.

\begin{tabular}{|c|c|c|c|}
\hline Parameters & Type 1 & Type 2 & Type 3 \\
\hline \multicolumn{4}{|c|}{ Central star } \\
\hline Effective temperature & \multicolumn{3}{|c|}{$5500 \mathrm{~K}$} \\
\hline Radius & \multicolumn{3}{|c|}{$7.5 R_{\odot}$} \\
\hline Mass & \multicolumn{3}{|c|}{$3.9 M_{\odot}$} \\
\hline \multicolumn{4}{|c|}{ Optically Thin Inner Disk } \\
\hline Inner radius (AU) & 1.8 & 0.80 & 0.52 \\
\hline$\Sigma \propto R^{\alpha}$ & -2.4 & -0.2 & -9 \\
\hline Total dust Mass $\left(M_{\oplus}\right)$ & $6.7 \times 10^{-4}$ & $4.7 \times 10^{-2}$ & $6.0 \times 10^{-5}$ \\
\hline The scale height & 0.3 & 0.24 & 0.39 \\
\hline \multicolumn{4}{|c|}{ Outer disk } \\
\hline$R_{\text {gap }}$ & & $45 \mathrm{AU}$ & \\
\hline Outer radius & & $500 \mathrm{AU}$ & \\
\hline Total Mass (gas+dust) & & $0.21 M_{\odot}$ & \\
\hline The scale height & & 0.15 & \\
\hline$\Sigma \propto R^{\alpha}$ & & -1 & \\
\hline
\end{tabular}

The distribution of dust in the gap is parameterized like a small independent disk. The outer radius $\left(R_{\text {gap }}\right)$, the inner disk radius $\left(R_{\mathrm{in}, \mathrm{gap}}\right)$, the total mass $\left(M_{\mathrm{in}, \mathrm{gap}}\right)$, and the power exponent $\left(\alpha_{\text {in }}\right)$, which assumes a distribution $\Sigma_{\text {in,dust }} \propto R^{\alpha_{\text {in }}}$ for the dust surface density $\left(\Sigma_{\text {in,dust }}\right)$ in the gap, are all left as free parameters. Hereafter, we refer to the inner disk and outer disk as the one in the gap and the one with radius $\geq R_{\text {gap }}$, respectively. We vary the free parameters in the models to search for the best-fit models. We find that the SED of GW Ori can be fitted very well with a model with $R_{\text {gap }}=45 \mathrm{AU}, R_{\text {in,gap }}=1.8 \mathrm{AU}$, and a total disk mass $\sim 0.21 M_{\odot}$. The best-fit SED is shown in Fig. 19. The parameters for the best-fit model are shown in Table 5 (Type 1).

We explore the parameter space of $R_{\text {gap }}$ by varying $R_{\text {gap }}$ and the other free parameters to fit the SED of GW Ori. In Fig. 20, we show the SEDs that are calculated from disk models with $R_{\text {gap }}=25$ and $55 \mathrm{AU}$, respectively. For clarity, we only show the SEDs at the wavelength range where different models are distinguishable from each other. A disk model with $R_{\text {gap }} \lesssim 25 \mathrm{AU}$ would have a stronger excess at $13-20 \mu \mathrm{m}$ than the observations, while one with $R_{\text {gap }} \gtrsim 55 \mathrm{AU}$ would produce weaker excess emission. Thus, we set $R_{\text {gap }}=25-55 \mathrm{AU}$ and take $45 \mathrm{AU}$ as the best value of $R_{\text {gap }}$. However, we must stress that our SED fits cannot provide strong constraints on $R_{\text {gap }}$, as well as other parameters, since the disk models have too many free parameters. The gap size would be affected by assuming different dust compositions and inner wall sizes. It is also affected if it is not pure gap but filled with non-axisymmetric structures caused by the companions. However, our attempt to fit the SED of GW Ori provides an important hint that disk models with gaps can reproduce the SED of GW Ori very well.

In our SED modeling, we only include two ingredients, the primary GW Ori A and a disk surrounding GW Ori. Recently, Berger et al. (2011) find that the brightness of GW Ori B can be $\sim 60 \%$ of the one of GW Ori A. Since GW Ori A and B are very close compared to the gap and the luminosity of GW Ori B has been added to that of GW Ori $\mathrm{A}$ in the modeling, it would not matter if we include one or two illuminating sources at the disk center. However, due to the interaction between the disk and the stellar system, the disk is most likely non-axisymmetric. The GW Ori $\mathrm{C}$ is much less luminous than GW Ori A and B (Berger et al. 2011), but it could also locally heat the disk and break the disk symmetry.

\subsubsection{Variations in the SED of GW Ori}

Mathieu et al. (1995) constructed the SED of GW Ori using the data from the literature (Rydgren \& Vrba 1983; Cohen \& Schwartz 1976; Cohen 1980, 1973), the IRAS survey, and their own observations at submillimeter and millimeter. The main difference between our SED and theirs is in the infrared bands. We use the infrared data from the 2MASS survey, WISE survey, and the Spitzer observations. In Fig. 21, we compare two SEDs at wavelengths $\lesssim 20 \mu \mathrm{m}$. We note that both SEDs are generally consistent with each other except in the wavelength range between the wavelengths of $1.6-3.5 \mu \mathrm{m}$. At $1.6-3.5 \mu \mathrm{m}$, the Mathieu's SED shows stronger excess emission than ours. In Fig. 21, we also show the observations of GW Ori collected from other works (Bouvier et al. 1988; Przygodda et al. 2003; Schegerer et al. 2009). In Bouvier et al. (1988), they monitored GW Ori in multiple bands during December 1984 and December 1986. In Fig. 21, we show the average photometry and the standard deviation in each band during each period. We note the data obtained in December 1986 show similar excess emissions at $1.6-3.5 \mu \mathrm{m}$ to those of Mathieu et al. (1995). Furthermore, the data obtained in December 1984 show strongest excess emissions at $1.6-3.5 \mu \mathrm{m}$ among all the observations. During the same period, the observation around $5 \mu \mathrm{m}$ show a dramatically decrease in flux and exhibits lowest excess among all the data. These temporal variations in near-infrared bands cannot be simply explained as variable extinctions, since their simultaneous data in optical bands do not show a significant difference. One promising explanation could be the change of inner-disk structure, since the fluxes of GW Ori at $1.6-3.5 \mu \mathrm{m}$ are dominated by the emission from the inner-disk region.

In Fig. 21, the SEDs of GW Ori in near- and mid-infrared bands can be classified into three types. The type 1 SED is referred to our constructed SED, the type 2 is for the Mathieu's, and the type 3 is the one observed in December 1984 from Bouvier et al. (1988). The detail modeling of the type 1 SED is described in Sect. 3.5.1, and their best-fit parameters are listed in Table 5. Here, we model the other two types. Given that we only have few multi-epoch observations at longer wavelengths $(\gtrsim 10 \mu \mathrm{m})$ and these data show no obvious variations, we fix the parameters for the outer disk in the modeling and use the best-fit parameters for the outer disk from fitting type 1 SED. We vary the parameters for the inner disk (see Sect. 3.5.1) to reproduce the other two types of SEDs. 

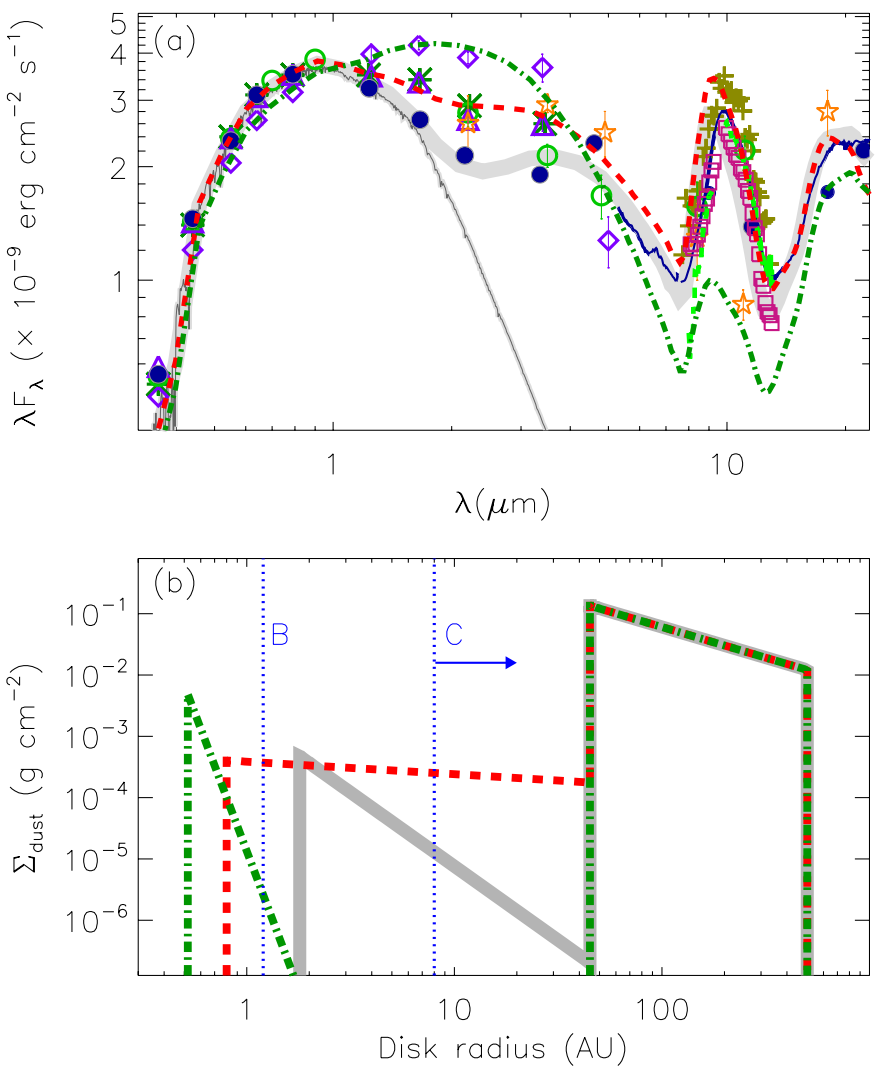

Fig. 21. a) SEDs of GW Ori constructed with the data observed at different epochs. Significant variations can be seen in the SED of GW Ori at near-infrared bands. Open stars mark the observations obtained at November 1971 (Cohen 1973). Open circles mark the data from November 1974 (Cohen \& Schwartz 1976). Asterisks display the data from December 1981 (Rydgren \& Vrba 1983). Open diamonds and triangles show the average photometry from December 1984 and December 1986, respectively (Bouvier et al. 1988). Plus symbols around $10 \mu \mathrm{m}$ show the data obtained on December 1978 (Cohen 1980), and the dashed lines around $10 \mu \mathrm{m}$ display the data obtained on December 2002 (Przygodda et al. 2003). The open boxes around $10 \mu \mathrm{m}$ show the data observed on March 2005 (Schegerer et al. 2009). Other symbols are the same as shown in Fig. 19. The thick gray curve shows calculated SED from Type 1; the dashed line for the SED from Type 2, and the dash-dotted line for the SED from Type 3. b) Surface density profiles for the dust in three model disks shown in panel a). The thick gray lines are for Type 1; the dashed lines are for Type 2, and the dashdotted lines are for Type 3 . The locations of two companions in GW Ori are also marked. The right-pointing arrow means that the separation for from GW Ori $\mathrm{C}$ to the primary is a lower limit.

Using the above simple models, we find that all the observed SEDs of GW Ori can be naturally explained by the change of the structure of the inner disk. In Fig. 21, we show the best-fit model SEDs for the three types of SEDs and the distribution of the surface densities for the disk models. The best-fit parameters are listed in Table 5. We note that a small amount of dust $\left(\sim 6 \times 10^{-5}-5 \times 10^{-2} M_{\oplus}\right)$ in the gap is enough to produce the observed excess emission at near- and mid-infrared bands. In each model the dust surface densities in the gap are reduced by several orders of magnitude, as compared with those at $R_{\text {gap }}$. However it is unknown whether the gas surface densities in the gap decrease by a similar factor to those of dust, since our SED modeling cannot provide any constraints on the gas material in the disk. From the type 1 to type 3 SED, the increase of excess emission in nearinfrared bands is interpreted as the decrease of $R_{\text {in }}$. The type 1 and type 2 models have similar excess emissions at wavelengths $\gtrsim 5 \mu \mathrm{m}$, while the type 3 model produces an abrupt drop of fluxes at wavelengths $\sim 5 \mu \mathrm{m}$ due to the dust material accumulating at radii $\sim 0.5 \mathrm{AU}$. Furthermore, the type 3 model produces a weaker silicate emission feature at $10 \mu \mathrm{m}$ compared with the other two models. Unfortunately, there are no simultaneous data around $10 \mu \mathrm{m}$ available, which makes this hypothetical disk structure hard to be tested.

Here, we simply assume that the dust in the gap is distributed in a disk structure. In reality, the distribution of dust material in the gap could be very complex due to the existence of two companions and the dust filtering in GW Ori (Artymowicz \& Lubow 1996; Günther \& Kley 2002; Rice et al. 2006; Zhu et al. 2012). In a binary system, simulations show that the material in the gap is mainly distributed in a set of two gas streams, which penetrate the gap and transfer the gas material from circumbinary disk to the central binary (Artymowicz \& Lubow 1996; Günther \& Kley 2002). In the simulations, the gas streams only account for $\sim 10 \%$ of the gap area and can carry at most $10^{-3}$ of the mass that would exist inside the gap for an unperturbed disk (Artymowicz \& Lubow 1996). The dynamic evolution of the gas streams can explain the variation in the SED. For GW Ori, a second companion does complicate this two-stream scenario. When including the effect of dust filtering, which can prevent large dust particles from crossing the gap in the disk, the scenario could be more complex. New simulations specified for GW Ori are extremely useful for understanding this system.

\section{Discussion: Disk evolution in binary/multiple systems}

\subsection{Accretion}

In December 1984 and December 1986, Bouvier et al. (1988) observed GW Ori in multiple bands. These data shows an obvious change in the SED of GW Ori in near-infrared bands (see Sect. 3.5.2 and Fig. 20). We explain the SED variations as the change of the dust distribution in the gap and find that the dust mass in the gap in December 1984 could be reduced by three orders of magnitude compared with the one in December 1986. From the $U$-band photometric data from Bouvier et al. (1988), the accretion rates of GW Ori at the two epochs are similar $\left(\sim 4 \times 10^{-7} M_{\odot} \mathrm{yr}^{-1}\right)$, However, the mean accretion rate of GW Ori in February 1984 is $\sim 7 \times 10^{-7} M_{\odot} \mathrm{yr}^{-1}$, which is about two times larger than the typical value $\left(\sim 4 \times 10^{-7} M_{\odot} \mathrm{yr}^{-1}\right.$, see Sect. 3.4.2). The latest observations of GW Ori before February 1984 were acquired on December 3, 1981 by Rydgren \& Vrba (1983), in which the $U$-band photometric data suggests an accretion rate $\sim 4 \times 10^{-7} M_{\odot} \mathrm{yr}^{-1}$. The enhancements of accretion for GW Ori lasted for less than 3 years and could accrete the material of less than $0.7 M_{\oplus}$. With an assumption of gas-to-dust ratio $\sim 100-1000$, the total accreted mass of dust is $\sim 7 \times 10^{-3}-7 \times 10^{-4} M_{\oplus}$, which is comparable to the dust masses in the gap, as suggested by our SED modeling (see Table 5). Therefore, it is possible that enhanced accretion in GW Ori can drain the material in the gap on a timescale of years, besides the gas-to-dust ratio in the gap is much larger than 1000 .

The simulations of Artymowicz \& Lubow (1996) show that a binary system can have pulsed accretion modulated by the orbital motion. For the low-eccentricity case like GW Ori, the theoretical works predict a smoothly enhanced accretion activity. Our observations show that the accretion rate of GW Ori is mostly constant and presents an accidental increase at orbital phases of $0.5-0.8$ by a factor of $2-3$. In Fig. 18, we compare 
the observations with the theoretical prediction. The simulation data are arbitrarily scaled to compare with the observations. We note the theory can explain the observational data in the orbital phases at which the enhanced accretion happens, as well as the level of the enhancement of accretion. However, as discussed in Sect. 3.4, we find the accretion rate of GW Ori is mostly constant over all the orbital phases of GW Ori B, which is inconsistent with the simulations from Artymowicz \& Lubow (1996). The simulations of binary systems also predict that low-mass companions accrete more masses than the primaries (Artymowicz \& Lubow 1996). However, as shown in Figs. 5 and 6, the EWs of accretion-related emission lines are modulated by the rotation of GW Ori A. This finding suggests that the accreting masses are mostly funneled onto GW Ori A, which also contradicts with the results in Artymowicz \& Lubow (1996). The spatial resolution of the simulations from Artymowicz \& Lubow (1996) may be too low to resolve the bridge-like stationary shock between the accretion disks of the two stellar components in binary systems, which has been found in the new hydrodynamical simulations (Fateeva et al. 2011; Bisikalo et al. 2012). Due to this collision, most of the material in the circumsecondary disk can be accreted onto the primary, which leads to higher rate of accretion onto the primary than onto the secondary (Fateeva et al. 2011). Our result is consistent with the new simulations.

\subsection{The disks in GW Ori}

\subsubsection{Dusty circumstellar disks in GW Ori}

Mathieu et al. (1991, 1995) successfully reproduced the SED of GW Ori using two-disk models with one circumstellar disk around GW Ori A and one circumbinary disk surrounding the GW Ori system. In their best-fit model, the circumstellar disk around GW Ori A has an inner radius of $0.025 \mathrm{AU}$ and an outer radius of $0.17 \mathrm{AU}$. The circumbinary disk starts from $3.3 \mathrm{AU}$ and extends to $110 \mathrm{AU}$. Inside the gap between the circumstellar disk and the circumbinary disk, they add a small amounts of dust to reproduce the strong silicate emission feature at $10 \mu \mathrm{m}$. Our disk models are slightly different from the Mathieu's since we do not include a dusty circumstellar disk around GW Ori A. Our arguments are follows.

The circumstellar disk around each component in a binary can be externally truncated by the tidal interaction with the companion, and the size of circumstellar disk is related to the eccentricity, the semi-major axis, and the mass ratio of the binary system (Pichardo et al. 2005). Assuming the orbital axis is aligned with the rotation axis of GW Ori A, the mass of GW Ori B is $\sim 0.3-0.7 M_{\odot}$ (see Sect. 3.2). According to the formula in Pichardo et al. (2005), the sizes of circumstellar disks around GW Ori A and B would be $\sim 0.3$, and $0.1 \mathrm{AU}$, respectively. Furthermore, the inner radius of a dusty disk can be determined by the evaporation of dust (Dullemond et al. 2001). For GW Ori A with $T_{\text {eff }}=5500 \mathrm{~K}$ and $L_{\star}=48 L_{\odot}$, the inner radius of its dusty disk is estimated to be $\sim 0.5 \mathrm{AU}$, assuming a dust evaporation temperature $\left(T_{\text {evap }}\right) \sim 1500 \mathrm{~K}$, according to the formula in Dullemond et al. (2001). Therefore, it is very likely that there is no dusty disk around GW Ori A. The stellar parameters of GW Ori B is very uncertain. For a $0.3-0.7 M_{\odot}$ PMS star at an age of $\sim 1 \mathrm{Myr}$, its $T_{\text {eff }}$ and $L_{\star}$ is expected to be $\sim 3400-4000 \mathrm{~K}$ and $\sim 0.7-1.7 L_{\odot}$, respectively (Siess et al. 2000). With these stellar parameters and $T_{\text {evap }}=1500 \mathrm{~K}$, the inner radius is $\sim 0.07 \mathrm{AU}$. If the orbital inclination of GW Ori B is much smaller $\left(\sim 10^{\circ}\right)$ as suggested in Berger et al. (2011), the mass of GW Ori B could be $1.4 M_{\odot}$ with $T_{\text {eff }} \sim 4500 \mathrm{~K}$ and $L_{\star} \sim 3.7 L_{\odot}$ (Siess et al. 2000), which gives an inner radius $\sim 0.13 \mathrm{AU}$. Thus, we may not expect there is a dusty disk around GW Ori B, too. For GW Ori C, it is very hard to characterize its disk properties, given that its stellar parameters are unknown. Thus, we neglect dusty circumstellar disks in our models. However, the gaseous circumstellar disk(s) must be harbored by at least one stellar member in GW Ori since GW Ori is still accreting (see Sect. 3.4).

\subsubsection{Dust filtration}

To reproduce the strong and sharp silicate feature at $10 \mu \mathrm{m}$, a small population of tiny dust particles needs to be included in the gap, which would be consistent with a dust filtration scenario in GW Ori. This effect has been proposed to explain the SEDs of transitional disk objects (TOs) and is supposed to occur in a disk with a gap where the gaseous pressure gradient can only let the dust grains be smaller than some critical size through the gap and trap the large dust particles, because of the dust radial drift (Rice et al. 2006). Recently, a direct evidence of dust trapping has been found in Oph IRS 48 with ALMA (van der Marel et al. 2013).

In Zhu et al. (2012), they discuss two effects to resist the dust radial drift: dust diffusion and amplified gas RV at the gap edge. When the dust diffusion is against the dust drift without regard for the amplified gas RV, Zhu et al. (2012) find a relation between the dust depletion factor $\left(\gamma / \gamma_{0}\right)$ and the gaseous gap depth $\left(\Sigma_{\mathrm{g}} / \Sigma_{\mathrm{g}, 0}\right)$, where $\gamma$ and $\Sigma_{\mathrm{g}}$ is the dust/gas mass ratio and the surface density inside the gap, respectively, and $\gamma_{0}$ and $\Sigma_{\mathrm{g}, 0}$ are these quantities at the gap edge. The relationship between $\gamma / \gamma_{0}$ and $\Sigma_{\mathrm{g}} / \Sigma_{\mathrm{g}, 0}$ depends on the dust particle density $\left(\rho_{\mathrm{p}}\right)$, dust particle radius $(s)$, gas surface density at the gap edge $\left(\Sigma_{\mathrm{g}, 0}\right)$, and disk viscosity parameter $(\alpha)$. Following the analytic approach in Zhu et al. (2012), we study the efficiency of dust filtration in the disk of GW Ori using the disk parameters (Type 1 in Table 5) of a best-fit model shown in Fig. 19. We take $\rho_{\mathrm{p}}=2.5 \mathrm{~g} / \mathrm{cm}^{-3}$ for the silicate grain, and $\alpha=0.01$. Figure 22a shows the relation between $\gamma / \gamma_{0}$ and $\Sigma_{\mathrm{g}} / \Sigma_{\mathrm{g}, 0}$ for dust particles with four sizes, $s=0.1,1,10$, and $100 \mu \mathrm{m}$. Note that a gaseous gap depth $\sim 0.04$ and 0.004 can efficiently $\left(\gamma / \gamma_{0}=10^{-3}\right)$ trap dust particles with sizes $100 \mu \mathrm{m}$ and $10 \mu \mathrm{m}$, respectively. To trap smaller dust particles, a deeper gaseous gap is required.

The dust drift velocity ( $\left.V_{\text {drift }}\right)$ needs to counteract not only the dust diffusion but also the amplified gas radial velocity $\left(V_{\mathrm{g}}\right)$ in the gap. In Fig. 22a, we do not consider the effect of amplified gas RV in the gap. Thus, the dust depletion factors are only the lower limits for dust particles at the gaseous gap depths. When the effect of amplified gas RV is included in the calculation, Zhu et al. (2012) built another relationship between ratios of $V_{\text {drift }}$ to $V_{\mathrm{g}}$ and gaseous gap depths. In Fig. 22b, we show ratios of $V_{\text {drift }}$ to $V_{\mathrm{g}}$ with respect to gaseous gap depths for dust particles with sizes of $0.1,1,10$, and $100 \mu \mathrm{m}$. When $V_{\text {drift }}$ is larger than $V_{\mathrm{g}}$, the dust particles are trapped. For a $10 \mu \mathrm{m}$-size dust grain, it can be trapped when $\Sigma_{\mathrm{g}} / \Sigma_{\mathrm{g}, 0} \sim 0.0003$. At a lower $\Sigma_{\mathrm{g}} / \Sigma_{\mathrm{g}, 0}$, smaller dust particles can be trapped. Our SED modeling can only provide the dust surface density in the disk. If we assume the gas-todust ratio is constant, $\Sigma_{\mathrm{g}} / \Sigma_{\mathrm{g}, 0}$ is $\sim 1.5 \times 10^{-6}$ for the disk model in Fig. 19. In this case, dust particles larger than $10 \mu \mathrm{m}$ can be efficiently filtered. However, it would be highly probable that the gas-to-dust ratio in the gap is higher than that in the outer disk if the dust filtration is occurring in the gap. Thus, the gaseous gap depth is expected to be more shallow than that from the SED modeling. Furthermore, our discussions here are tentative since they are based on the disk parameters from the SED modeling, which are very uncertain. 


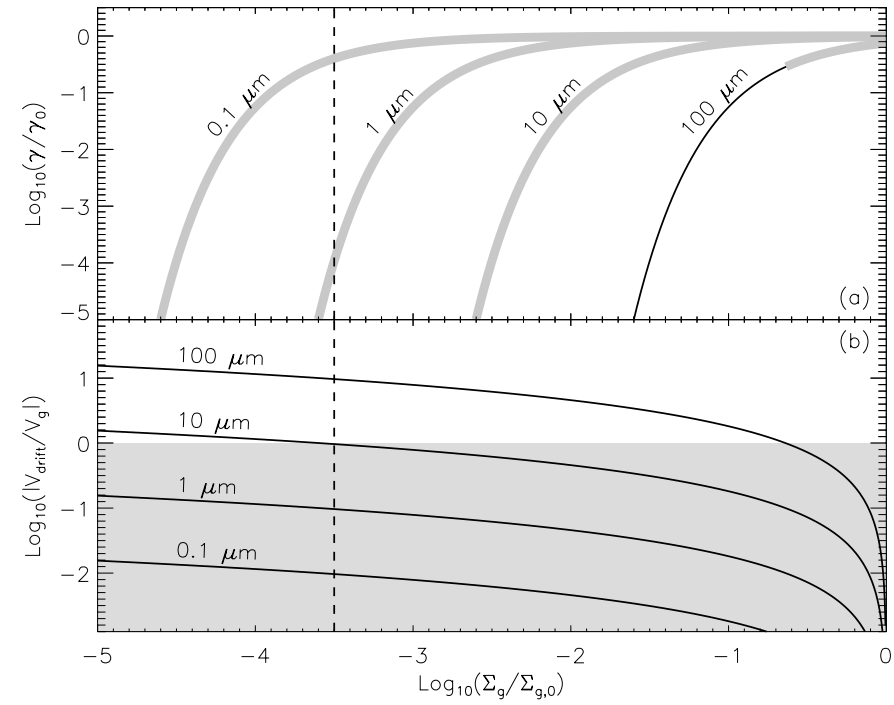

Fig. 22. a) Dust depletion factor due to the dust filtration by gap edges as a function of the gap depth for 4 grain sizes of dust, which include $0.1,1,10$, and $100 \mu \mathrm{m}$. The dust depletion factor is calculated assuming that the dust diffusion balances the dust drift. For each grain size, the thick gray line shows the regime where the gas velocity amplification at the gap edge dominates (see panel b)). In this case, the dust depletion factor is only the lower limit at the gap depth. The dashed line marks the gap depth at which the dust particle with size $\geq 10 \mu \mathrm{m}$ can be trapped because the dust drift velocity is larger than the gas velocity (see panel b)). b) The ratio of the dust drift velocity to the gas velocity with respect to the gap depth for the dust grains with different sizes. In the calculation, we consider the amplification of gas velocity in the gap and neglect the effect of dust diffusion. The gray-filled region marks, where the ratio is smaller than 1 and dust particles can pass through the gap. The dust grains will be prevented from crossing the gap when the ratio is larger than 1 . The dashed line is same as in panel a). The figure is made using the equations in Zhu et al. (2012).

\subsubsection{Gravitational instability}

The strong continuum emissions at submillimeter and millimeter wavelength suggest that GW Ori system is harboring a massive disk (Mathieu et al. 1995). Such a disk may suffer from the gravitational instability. A criterion to judge the gravitational instability in a disk is given by the Toomre parameter $(Q$; Toomre 1964):

$Q=\frac{c_{\mathrm{S}} \Omega}{2 \pi G \Sigma}$,

where $c_{\mathrm{S}}$ is the sound speed, $\Omega$ is the Keplerian angular velocity, $G$ is the gravitational constant, and $\Sigma$ is the surface density of disk. The instability occurs in the disk when $Q<1$, in which the disk is sufficiently cool, or massive, and when $Q>1$, in which the disk is stable and not fragmented. We calculate the $Q$ value for the circumbinary disk of GW Ori using the disk parameters (Type 1 in Table 5) for the model shown in Fig. 19. The Toomre parameter decreases with the disk radius but is always larger than 1, indicating that the disk of GW Ori is stable at all radii. The other two types of disk models in Fig. 21 and Table 5 are only different from the type 1 model in the inner-disk structure $(<45 \mathrm{AU})$, and are also stable at all the disk radii.

\subsection{Gap sizes and binary separations}

The study toward several star-forming regions, including Taurus, Cha I, and Ophiuchus, reveals about $2 / 3$ of close binaries

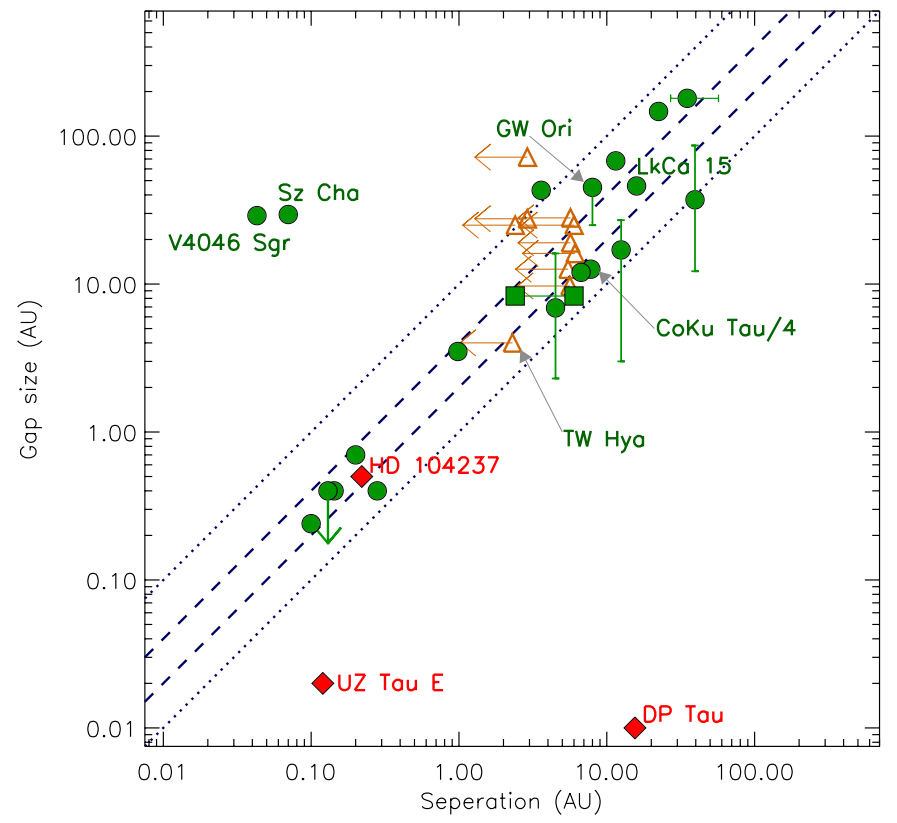

Fig. 23. Gap size of a disk surrounding a binary/multiple stellar system vs. separation from the companion to the primary. The filled circles show the disks with gaps, and the filled diamonds are for the disks without gaps. The filled boxes connected by a solid line are for the source FL Cha of which two possible separations are given in Cieza et al. (2013). The open triangles show the disks with gaps but without known companions. The separations for these disks are the detection limits at given mass ratios of companions to primaries (see Appendix A). The left-pointing arrows are used to indicate that it could be still possible that there are companions at smaller distances than the detection limits. The dotted lines mark the 1:1 and 10:1 relation between gap sizes and separations. The dashed lines show the predicted relation between gap sizes and separations from Pichardo et al. (2008) with $e=0$ and 1.

( $\lesssim 40 \mathrm{AU}$ ) have dispersed their disks at ages $<1 \mathrm{Myr}$, thus providing important constraints on the timescale of planet formation in such systems (Kraus et al. 2012). A fast dissipation of disks in close binaries is expected from theoretical works (Lin \& Papaloizou 1993). In the inner region of a circumbinary disk, a gap quickly forms and isolates the circumstellar disks from the circumbinary disk. Both disks evolve independently. Based on the calculations of Artymowicz \& Lubow (1994), a circumbinary disk can be truncated inside at $R_{\text {gap }} \sim 1.8 a-2.6 a$, where $a$ is the orbital semi-major axis of a binary. Pichardo et al. (2008) find the similar result. They run the simulations with mass ratios $0.1 \leq q \leq 0.9$ and eccentricity $0 \leq e \leq 0.9$ and find $R_{\text {gap }} \approx 1.93 a\left(1+1.01 e^{0.32}\right)[q(1-q)]^{0.043}$. For GW Ori, the circumbinary disk is probably truncated by GW Ori C, which gives $R_{\text {gap }} \sim 2 a-4 a$ at extreme cases of $e=0$ and 1, by assuming $q>0.1$. Given a projected separation of $\sim 8 \mathrm{AU}$ between GW Ori A and GW Ori C, $R_{\text {gap }}$ is around 16-32 AU, which is comparable to the result (25-55 AU) from our SED modeling.

In Fig. 23, we collect a sample of binary/multiple systems with circumbinary disks and show their gap sizes with respect to the separations $(a)$ between the companions and the primaries. The detail information for each binary is described in Appendix A. Here, the separations shown in Fig. 23 are mostly projected values and thus present lower limits of the real values. Among the sources in Fig. 23, three systems, including UZ Tau E, DP Tau, and HD 104237, may be harboring circumbinary disks without any evidences of gaps. The systems UZ Tau E and DP Tau could be too young to clear out their inner disks. The object HD 104237 is an A-type hot young star. Its inner radius 
of a disk set by the dust evaporation is around $0.5 \mathrm{AU}$, comparable to the one that are supposed to be truncated by the companion. In Fig. 23, we also see that two systems, V4046 Sgr and $\mathrm{Sz}$ Cha, which have very small separations $(<0.1 \mathrm{AU})$ but are harboring disks with much larger gaps than the expectation from the theory (Artymowicz \& Lubow 1994; Pichardo et al. 2008). The accretion rates of these sources are typically lower $\left(2 \times 10^{-9}-6 \times 10^{-10} M_{\odot} \mathrm{yr}^{-1}\right.$, Donati et al. 2011; Espaillat et al. 2011), and the photoevaportion process is expected to take effect in the disk dissipation and clear out the inner regions of disks (Alexander et al. 2006; Owen et al. 2011). However, it cannot be excluded that in these systems there are "unseen" companions at separations large enough to create the big gaps in the disks.

To test the significance of the correlation between the gap sizes and separations in Fig. 23, we apply a Kendall $\tau$ test. The Kendall $\tau$ test yields $\tau=0.52$ and $p=3 \times 10^{-4}$ for all the sources in Fig. 23, suggesting the gap sizes and separations are clearly correlated. If we exclude the "outliers" discussed in the above paragraph, the correlation between the values are more significant with $\tau=0.81$ and $p=1 \times 10^{-6}$ from the Kendall $\tau$ test. In Fig. 23, we also show the relation between the gap sizes and the separations predicted by Pichardo et al. (2008) for $e=0$ and 1. We note the sources in Fig. 23 follow the relations in general but are distributed within a broad "band" (a large scatter). This can be due to the large uncertainties in both the gap sizes and the separations, since the separations are mostly projected ones and the gap sizes are usually estimated from SED modeling (see Appendix A). In Fig. 23, about 56\% of systems have values of $1<R_{\mathrm{gap}} / a<4$ and the median value of $R_{\mathrm{gap}} / a$ is around 3, which is consistent with the theoretical prediction (Artymowicz \& Lubow 1994; Pichardo et al. 2008).

In Fig. 23, we also show a collection of transition objects (TOs). These sources have been observed with high-resolution imaging and do not show any stellar or substellar companions at separations less than several AUs, although they are known to have gaps in the disks. We use the detection limits of these observations as the upper limits of separations between "unseen" companions and the central stars. For these TOs, some other physical mechanisms, such as photoevaportion, may be responsible for the clearing of their inner disks. However, we note that most of these TOs are still located within the broad "band", where the binary systems are distributed in the figure. It could be still possible that there are "unseen" companions at separations smaller than the detection limits of the observations.

\section{Summary}

We have studied the triple system GW Ori with a special focus on the accretion properties and disk properties, using a combination of available archive data and our own high-resolution spectroscopic data from FEROS and HARPS as observed during 2007 and 2010. We summarize our main results as follows:

- By comparing the FEROS spectra with the synthetical spectra, we classify GW Ori A as G8 type. The mass and age of GW Ori is estimated to be $\sim 3.9 M_{\odot}$ and is less than $1 \mathrm{Myr}$, which points out that GW Ori A could be at a rapid transitional phase to a Herbig Be star.

- We analyze the RVs of the spectroscopic data of GW Ori and confirm a companion around GW Ori with a period of $\sim 242$ days and an orbital semi-major axis of $\sim 1 \mathrm{AU}$. The residual RVs from the orbital solution and the EWs of accretion-related emission lines show periodic variations during short terms (5-6.7 days). We explain that they are from the modulation with stellar rotation of GW Ori A.
- The variance profiles of the $\mathrm{H} \alpha$ and $\mathrm{H} \beta$ lines of GW Ori present blue-shifted peaks and are featureless on the red side. The autocorrelation matrices for the $\mathrm{H} \alpha$ and $\mathrm{H} \beta$ lines show a clear correlation between the blue $\left(-300-0 \mathrm{~km} \mathrm{~s}^{-1}\right)$ and the red $\left(0-200 \mathrm{~km} \mathrm{~s}^{-1}\right)$ sides of profiles. The $\mathrm{H} \alpha$ and $\mathrm{H} \beta$ line profiles can be decomposed very well by two emission components and one blue-shifted absorption component. The absorption component can be attributed to the disk wind launched at a disk radius near the orbit of GW Ori B. The strength of the disk wind can be modulated by the orbital motion of GW Ori B.

- We investigate the accretion behavior of GW Ori using the accretion-related emission lines and $U$-band photometry. Our results contradict the simulations of Artymowicz \& Lubow (1996) at two points: (1) the accreted material of GW Ori appears to be mostly funneled onto the primary GW Ori A; (2) the accretion rates of GW Ori are mostly constant and are only occasionally enhanced by a factor of 2-3 within the orbital phases of $0.5-0.8$. At Point (1), our result is consistent with the new simulations from Fateeva et al. (2011).

- We reproduce the SED of GW Ori using disk models with gaps sized 25-55 AU. The gap sizes in our models are much larger than the previous result (3.3 AU) in Mathieu et al. (1995), which are comparable to the truncation of the inner disk radius (16-32 AU), as expected from the simulations, considering the second companion GW Ori C. In the gap, only a small amount of tiny dust particles are added to produce the near-infrared excess emission and the strong and sharp silicate emission feature at $10 \mu \mathrm{m}$. Using the best-fit parameters of the disk models, we study the efficiency of dust filtration in the disk of GW Ori and find that dust grains larger than $10 \mu \mathrm{m}$ can be efficiently trapped in the outer disk.

- We find that GW Ori shows dramatic changes in its SED in near-infrared bands, which implies a major readjustment of the inner disk on timescales of $\sim 20 \mathrm{yr}$. We discuss the possibility that the material in the gap is drained by the accretion in GW Ori and find that the timescale to exhaust the material in the gap via accretion can be several years only if the gasto-dust ratio in the gap is $\sim 100-1000$, which can explain the rapid variation in the SED of GW Ori in near-infrared bands.

- A sample of binary/multiple systems collected in the literature exhibits a strong positive correlation between their gap sizes and the separations of companions to the primaries, which is consistent with the expectation from the theory.

Acknowledgements. We thank the referee, Dr. S. Lamzin, for the positive and useful comments. We want to acknowledge C. Dullemond for providing us with his RADMC code. M.F. acknowledges support of the action "Proyectos de Investigación fundamental no orientada", grant number AYA2012-35008. ASA support of the Spanish MICINN/MINECO "Ramón y Cajal" program, grant number RYC-2010-06164, and the action "Proyectos de Investigación fundamental no orientada", grant number AYA2012-35008. V.R. was partially supported by the Bayerischen Gleichstellungsförderung $(B G F)$. This research has made use of the SIMBAD database, operated at CDS, Strasbourg, France. This publication makes use of data products from the Two Micron All Sky Survey, which is a joint project of the University of Massachusetts and the Infrared Processing and Analysis Center/California Institute of Technology, funded by the National Aeronautics and Space Administration and the National Science Foundation. This publication makes use of data products from the Widefield Infrared Survey Explorer, which is a joint project of the University of California, Los Angeles, and the Jet Propulsion Laboratory/California Institute of Technology, funded by the National Aeronautics and Space Administration. This research is based on observations with AKARI, a JAXA project with the participation of ESA. This work is in part based on observations made with the Spitzer Space Telescope, which is operated by the Jet Propulsion Laboratory, California Institute of Technology under a contract with NASA. 
Table A.1. Parameters for young systems with companions/planets.

\begin{tabular}{|c|c|c|c|c|c|c|c|}
\hline \multicolumn{8}{|c|}{ With Gap } \\
\hline Name & Mass ratio & $\begin{array}{l}\text { Separation } \\
\text { (AU) }\end{array}$ & Method1 & Eccentricity & $\begin{array}{l}\text { Gap Size } \\
(\mathrm{AU})\end{array}$ & Method2 & Reference \\
\hline CoKu Tau/4 & 0.85 & 7.8 & Image & - & 12.6 & SED Fit & 1,2 \\
\hline $\mathrm{T}$ Cha & - & 6.7 & Image & - & 12 & Mid-IR Int & 3,4 \\
\hline V4046 Sgr & 0.96 & $0.04^{a}$ & Spec, D & $<0.01$ & 29 & MM Int & 5,6 \\
\hline AK Sco & 0.987 & 0.14 & Spec, D & 0.47 & 0.4 & SED Fit & 7 \\
\hline GG Tau A & 0.9 & $35_{-8}^{+22 b}$ & Image & $0.3 \pm 0.2$ & 180 & MM Int & $8,9,10$ \\
\hline CS Cha & - & $\sim 3.6^{c}$ & Spec, $\mathrm{S}$ & - & 43 & SED Fit & $11,12,13$ \\
\hline HD $98800 \mathrm{~B}$ & 0.83 & 0.98 & Spec, D & 0.78 & 3.5 & SED Fit & $14,15,16$ \\
\hline DQ Tau & 0.97 & 0.13 & Spec, D & 0.556 & $\lesssim 0.4$ & SED Fit & 17,18 \\
\hline $162814-2427$ & 0.92 & 0.28 & Spec, D & 0.48 & 0.4 & SED Fit & 19,20 \\
\hline $162819-2423 \mathrm{~S}$ & $\gtrsim 0.26$ & 0.1 & Spec, S & 0.41 & 0.24 & SED Fit & 19,20 \\
\hline FL Cha & - & $2.4 / 6$ & Image & - & 8.3 & SED Fit & 21 \\
\hline $\mathrm{LkH} \alpha 330$ & - & $10-13$ & Image & - & 68 & MM Int & 22,23 \\
\hline $\mathrm{T} 21$ & - & 22.4 & Image & - & 146.7 & SED Fit & 24,25 \\
\hline DF Tau & 0.75 & $12.5^{d}$ & Image & 0.51 & $17_{-14}^{+10}$ & SED Fit & $26,27,28$ \\
\hline MHO 3 & 0.5 & 4.5 & Image & - & $6.9_{-4.6}^{+9.2}$ & SED Fit & 29,30 \\
\hline CHX22 & - & 39.5 & Image & - & $37.1_{-24.9}^{+49.3}$ & SED Fit & 24,30 \\
\hline ST 34 & $\sim 1$ & $\sim 0.2^{e}$ & Spec, D & - & 0.7 & SED Fit & 31,32 \\
\hline Sz Cha & & $\sim 0.07^{f}$ & Spec, S & - & 29.5 & SED Fit & 33,30 \\
\hline LkCa 15 & $\sim 0.006$ & $\sim 15.9$ & Image & - & 50 & MM Int & 34,35 \\
\hline \multicolumn{8}{|c|}{ Without Gap } \\
\hline Name & Mass ratio & $\begin{array}{l}\text { Separation } \\
\text { (AU) }\end{array}$ & Method1 & Eccentricity & $\begin{array}{l}R_{\text {in }} \\
(\mathrm{AU})\end{array}$ & Method2 & Reference \\
\hline HD 104237 & 0.64 & $0.22^{g}$ & Spec, $S$ & 0.66 & 0.5 & SED Fit & $36,37,38$ \\
\hline UZ Tau E & 0.30 & 0.124 & Spec, D & 0.33 & $\sim 0.02$ & $R_{\text {sub }}$ & 39,40 \\
\hline DP Tau & 0.74 & 15.5 & Image & - & $\sim 0.01$ & $R_{\text {sub }}$ & 29,41 \\
\hline \multicolumn{8}{|c|}{ TOs without known companions and their detection limit } \\
\hline Name & Mass ratio & $\begin{array}{c}\text { Separation } \\
\text { (Detection limit, AU) }\end{array}$ & Method1 & Eccentricity & $\begin{array}{l}\text { Gap Size } \\
(\mathrm{AU})\end{array}$ & Method2 & Reference \\
\hline GM Aur & 0.01 & 5.7 & Image & - & 28 & MM Int & 29,22 \\
\hline UX Tau A & 0.01 & 6 & Image & - & 25 & MM Int & 29,22 \\
\hline RY Tau & 0.01 & 2.9 & Image & - & 27.6 & SED Fit & 29,30 \\
\hline DM Tau & 0.02 & 5.7 & Image & - & 19 & MM Int & 29,22 \\
\hline GK Tau & 0.02 & 5.6 & Image & - & 9.7 & SED Fit & 29,22 \\
\hline HK Tau & 0.02 & 6.1 & Image & - & 16.1 & SED Fit & 29,30 \\
\hline RX J1604.3-2130 & 0.02 & 3 & Image & - & 72 & MM Int & 42,43 \\
\hline IP Tau & 0.03 & 5.5 & Image & - & 12.6 & SED Fit & 29,30 \\
\hline RX J1633.9-2442 & $\sim 0.02$ & 2.4 & Image & - & $\sim 25$ & MMT Int & 44 \\
\hline TW Hya & - & 2.3 & Image & - & 4 & SED Fit & 45,46 \\
\hline
\end{tabular}

Notes. Column 3: if it is not specified, the separation is projected distance between two stellar components when the binary is detected with imaging, and is minimum semi-major of the orbit when it is detected with spectroscopy. Column 4: the methods with which the binaries are detected: Image for imaging, Spec for spectroscopy, D for double lines, and S for single line. Column 4: the methods with which the gap sizes are estimated, including fitting the SED (SED Fit), mid-infrared interferometry (Mid-IR Int), millimeter interferometry (MM Int). For the full disks (UZ Tau E and DP Tau), the inner radius of the disks are determined by the evaporation of dust $\left(R_{\text {sub }}\right){ }^{(a)}$ sum of semi-majors of the orbits for the two stellar components with an assumption of inclination $35^{\circ} ;{ }^{(b, d)}$ the orbital semi-major from fitting the orbit. c: roughly estimated using the orbit period 2482 day; ${ }^{(e)}$ roughly estimated assuming the system is edge-on; ${ }^{(f)}$ roughly estimated using the orbit period of 5 days; ${ }^{(g)}$ the semi-major of the orbit assuming an inclination $17^{\circ}$.

References. 1). Ireland \& Kraus (2008); 2). Nagel et al. (2010); 3). Huélamo et al. (2011); 4). Olofsson et al. (2013); 5). Stempels \& Gahm (2004); 6). Rosenfeld et al. (2013); 7). Alencar et al. (2003); 8). White et al. (1999); 9). McCabe et al. (2002); 10). Guilloteau et al. (1999); 11). Guenther et al. (2007); 12). Espaillat et al. (2007); 13). Nagel et al. (2012); 14). Torres et al. (1995); 15). Boden et al. (2005); 16). Andrews et al. (2010); 17). Mathieu et al. (1997); 18). Boden et al. (2009); 19). Mathieu et al. (1989); 20). Jensen \& Mathieu (1997); 21). Cieza et al. (2013); 22). Andrews et al. (2011); 23). Isella et al. (2013); 24). Lafrenière et al. (2008); 25). Kim et al. (2009); 26). White \& Ghez (2001); 27). Marsh \& Mahoney (1993); 28). Tamazian et al. (2002); 29). Kraus et al. (2011) 30). Kim et al. (2013); 31). White \& Hillenbrand (2005); 32). Hartmann et al. (2005); 33). Reipurth et al. (2002); 34). Kraus \& Ireland (2012); 35). Piétu et al. (2006); 36). Böhm et al. (2004); 37). Tatulli et al. (2007); 38). Garcia et al. (2013); 39). Jensen et al. (2007); 40). Jensen et al. (1996); 41). Furlan et al. (2006); 42). Kraus et al. (2008); 43). Mathews et al. (2012); 44). Cieza et al. (2012); 45). Calvet et al. (2002); 46). Brandeker et al. (2003).

\section{Appendix A: Young binary systems}

We collect a sample of binary systems with disks and present them in Table A.1. We list the companion/primary mass ratios, the separations, then eccentricities of the binaries, the gap sizes of the disks, and the methods using which the gap sizes are derived. In the table, the young star LkCa 15 with a possible planet is also listed. As a comparison, we also list three spectroscopic binaries without sign of gaps in their disks in Table A.1.

In Table A.1, ten TOs are listed. Kraus et al. (2008, 2011), and Cieza et al. (2012) have imaged these TOs with a high spatial 
resolution and found no companions. The separations of these TOs are on a lower limit of distances from the central stars, where the observations can detect companions at given companion/primary mass ratios.

\section{References}

Alencar, S. H. P., Melo, C. H. F., Dullemond, C. P., et al. 2003, A\&A, 409, 1037 Alexander, R. D., Clarke, C. J., \& Pringle, J. E. 2006, MNRAS, 369, 229 Andrews, S. M., Czekala, I., Wilner, D. J., et al. 2010, ApJ, 710, 462 Andrews, S. M., Wilner, D. J., Espaillat, C., et al. 2011, ApJ, 732, 42 Artymowicz, P., \& Lubow, S. H. 1994, ApJ, 421, 651 Artymowicz, P., \& Lubow, S. H. 1996, ApJ, 467, L77

Basri, G., Johns-Krull, C. M., \& Mathieu, R. D. 1997, AJ, 114, 781

Berger, J.-P., Monnier, J. D., Millan-Gabet, R., et al. 2011, A\&A, 529, L1 Beristain, G., Edwards, S., \& Kwan, J. 2001, ApJ, 551, 1037

Bisikalo, D. V., Dodin, A. V., Kaigorodov, P. V., et al. 2012, Astron. Rep., 56, 686

Boden, A. F., Sargent, A. I., Akeson, R. L., et al. 2005, ApJ, 635, 442 Boden, A. F., Akeson, R. L., Sargent, A. I., et al. 2009, ApJ, 696, L111 Böhm, T., Catala, C., Balona, L., \& Carter, B. 2004, A\&A, 427, 907 Bouvier, J. 1990, AJ, 99, 946

Bouvier, J., Bertout, C., \& Bouchet, P. 1988, A\&AS, 75, 1

Bouvier, J., Alencar, S. H. P., Harries, T. J., Johns-Krull, C. M., \& Romanova, M. M. 2007, Protostars and Planets V (Tucson: University of Arizona press), 479

Bouvier, J., Grankin, K. N., Alencar, S. H. P., et al. 2003, A\&A, 409, 169

Bouwman, J., Lawson, W. A., Dominik, C., et al. 2006, ApJ, 653, L57 Bouwman, J., Henning, T., Hillenbrand, L. A., et al. 2008, ApJ, 683, 479 Brandeker, A., Jayawardhana, R., \& Najita, J. 2003, AJ, 126, 2009 Calvet, N., \& Gullbring, E. 1998, ApJ, 509, 802

Calvet, N., D'Alessio, P., Hartmann, L., et al. 2002, ApJ, 568, 1008

Calvet, N., Muzerolle, J., Briceño, C., et al. 2004, AJ, 128, 1294

Cardelli, J. A., Clayton, G. C., \& Mathis, J. S. 1989, ApJ, 345, 245

Cieza, L. A., Padgett, D. L., Allen, L. E., et al. 2009, ApJ, 696, L84

Cieza, L. A., Mathews, G. S., Williams, J. P., et al. 2012, ApJ, 752, 75

Cieza, L. A., Lacour, S., Schreiber, M. R., et al. 2013, ApJ, 762, L12

Coelho, P., Barbuy, B., Meléndez, J., Schiavon, R. P., \& Castilho, B. V. 2005, A\&A, 443, 735

Cohen, M. 1973, MNRAS, 161, 97

Cohen, M. 1980, MNRAS, 191, 499

Cohen, M., \& Schwartz, R. D. 1976, MNRAS, 174, 137

Donati, J.-F., Gregory, S. G., Montmerle, T., et al. 2011, MNRAS, 417, 1747

Dotter, A., Chaboyer, B., Jevremović, D., et al. 2008, ApJS, 178, 89

Dullemond, C. P., \& Dominik, C. 2004, A\&A, 417, 159

Dullemond, C. P., Dominik, C., \& Natta, A. 2001, ApJ, 560, 957

Duquennoy, A., \& Mayor, M. 1991, A\&A, 248, 485

Espaillat, C., Calvet, N., D’Alessio, P., et al. 2007, ApJ, 664, L111

Espaillat, C., Furlan, E., D’Alessio, P., et al. 2011, ApJ, 728, 49

Fang, M., van Boekel, R., Wang, W., et al. 2009, A\&A, 504, 461

Fang, M., van Boekel, R., King, R. R., et al. 2012, A\&A, 539, A119

Fang, M., Kim, J. S., van Boekel, R., et al. 2013a, ApJS, 207, 5

Fang, M., van Boekel, R., Bouwman, J., et al. 2013b, A\&A, 549, A15

Fateeva, A. M., Bisikalo, D. V., Kaygorodov, P. V., \& Sytov, A. Y. 2011, Ap\&SS, 335,125

Fedele, D., van den Ancker, M. E., Henning, T., Jayawardhana, R., \& Oliveira, J. M. 2010, A\&A, 510, A72

Fischer, D. A., \& Marcy, G. W. 1992, ApJ, 396, 178

Furlan, E., Hartmann, L., Calvet, N., et al. 2006, ApJS, 165, 568

Garcia, P. J. V., Benisty, M., Dougados, C., et al. 2013, MNRAS, 430, 1839

Ghez, A. M., Neugebauer, G., \& Matthews, K. 1993, AJ, 106, 2005

Ghez, A. M., McCarthy, D. W., Patience, J. L., \& Beck, T. L. 1997, ApJ, 481, 378

Grankin, K. N., Melnikov, S. Y., Bouvier, J., Herbst, W., \& Shevchenko, V. S. 2007, A\&A, 461, 183

Guenther, E. W., Esposito, M., Mundt, R., et al. 2007, A\&A, 467, 1147

Guilloteau, S., Dutrey, A., \& Simon, M. 1999, A\&A, 348, 570

Gullbring, E., Petrov, P. P., Ilyin, I., et al. 1996, A\&A, 314, 835

Gullbring, E., Hartmann, L., Briceno, C., \& Calvet, N. 1998, ApJ, 492, 323

Günther, R., \& Kley, W. 2002, A\&A, 387, 550

Haisch, Jr., K. E., Lada, E. A., \& Lada, C. J. 2001, ApJ, 553, L153

Hartigan, P., Hartmann, L., Kenyon, S., Hewett, R., \& Stauffer, J. 1989, ApJS 70,899

Hartmann, L., Calvet, N., Gullbring, E., \& D’Alessio, P. 1998, ApJ, 495, 385

Hartmann, L., Calvet, N., Watson, D. M., et al. 2005, ApJ, 628, L147
Heckmann, O. 1975, AGK 3. Star catalogue of positions and proper motions north of -2.5 deg declination ed. W. Dieckvoss

Herbig, G. H. 1977, ApJ, 214, 747

Herczeg, G. J., \& Hillenbrand, L. A. 2008, ApJ, 681, 594

Hernández, J., Hartmann, L., Megeath, T., et al. 2007, ApJ, 662, 1067

Hillenbrand, L. A. 2002 [arXiv:astro-ph/0210520]

Houck, J. R., Roellig, T. L., van Cleve, J., et al. 2004, ApJS, 154, 18

Huélamo, N., Lacour, S., Tuthill, P., et al. 2011, A\&A, 528, L7

Ireland, M. J., \& Kraus, A. L. 2008, ApJ, 678, L59

Isella, A., Pérez, L. M., Carpenter, J. M., et al. 2013, ApJ, 775, 30

Ishihara, D., Onaka, T., Kataza, H., et al. 2010, A\&A, 514, A1+

Janson, M., Hormuth, F., Bergfors, C., et al. 2012, ApJ, 754, 44

Jensen, E. L. N., \& Mathieu, R. D. 1997, AJ, 114, 301

Jensen, E. L. N., Koerner, D. W., \& Mathieu, R. D. 1996, AJ, 111, 2431

Jensen, E. L. N., Dhital, S., Stassun, K. G., et al. 2007, AJ, 134, 241

Johns, C. M., \& Basri, G. 1995a, AJ, 109, 2800

Johns, C. M., \& Basri, G. 1995b, ApJ, 449, 341

Joy, A. H. 1949, ApJ, 110, 424

Kaufer, A., Stahl, O., Tubbesing, S., et al. 1999, The Messenger, 95, 8

Kenyon, S. J., \& Hartmann, L. 1995, ApJS, 101, 117

Kim, K. H., Watson, D. M., Manoj, P., et al. 2009, ApJ, 700, 1017

Kim, K. H., Watson, D. M., Manoj, P., et al. 2013, ApJ, 769, 149

Kraus, A. L., \& Ireland, M. J. 2012, ApJ, 745, 5

Kraus, A. L., Ireland, M. J., Martinache, F., \& Lloyd, J. P. 2008, ApJ, 679, 762

Kraus, A. L., Ireland, M. J., Martinache, F., \& Hillenbrand, L. A. 2011, ApJ, 731,8

Kraus, A. L., Ireland, M. J., Hillenbrand, L. A., \& Martinache, F. 2012, ApJ, 745,19

Kurosawa, R., \& Romanova, M. M. 2013, MNRAS, 431, 2673

Lafrenière, D., Jayawardhana, R., Brandeker, A., Ahmic, M., \& van Kerkwijk, M. H. 2008, ApJ, 683, 844

Landolt, A. U. 1983, AJ, 88, 439

Leinert, C., Zinnecker, H., Weitzel, N., et al. 1993, A\&A, 278, 129

Lin, D. N. C., \& Papaloizou, J. C. B. 1993, in Protostars and Planets III, eds. E. H. Levy, \& J. I. Lunine, 749

Lubow, S. H., \& Artymowicz, P. 2000, Protostars and Planets IV (TUCSON: University of Arizona press), 731

Marsh, K. A., \& Mahoney, M. J. 1993, ApJ, 405, L71

Martín, E. L., Magazzù, A., Delfosse, X., \& Mathieu, R. D. 2005, A\&A, 429, 939

Mathews, G. S., Williams, J. P., \& Ménard, F. 2012, ApJ, 753, 59

Mathieu, R. D., Walter, F. M., \& Myers, P. C. 1989, AJ, 98, 987

Mathieu, R. D., Adams, F. C., \& Latham, D. W. 1991, AJ, 101, 2184

Mathieu, R. D., Adams, F. C., Fuller, G. A., et al. 1995, AJ, 109, 2655

Mathieu, R. D., Stassun, K., Basri, G., et al. 1997, AJ, 113, 1841

Mayor, M., Pepe, F., Queloz, D., et al. 2003, The Messenger, 114, 20

McCabe, C., Duchêne, G., \& Ghez, A. M. 2002, ApJ, 575, 974

Mendigutía, I., Calvet, N., Montesinos, B., et al. 2011, A\&A, 535, A99

Mohanty, S., Jayawardhana, R., \& Basri, G. 2005, ApJ, 626, 498

Müller, A., van den Ancker, M. E., Launhardt, R., et al. 2011, A\&A, 530, A85

Müller, A., Roccatagliata, V., Henning, T., et al. 2013, A\&A, 556, A3

Murdin, P., \& Penston, M. V. 1977, MNRAS, 181, 657

Muzerolle, J., Hartmann, L., \& Calvet, N. 1998, AJ, 116, 455

Nagel, E., D'Alessio, P., Calvet, N., et al. 2010, ApJ, 708, 38

Nagel, E., Espaillat, C., D’Alessio, P., \& Calvet, N. 2012, ApJ, 747, 139

Olofsson, J., Benisty, M., Le Bouquin, J.-B., et al. 2013, A\&A, 552, A4

Owen, J. E., Ercolano, B., \& Clarke, C. J. 2011, MNRAS, 412, 13

Pecaut, M. J., \& Mamajek, E. E. 2013, ApJS, 208, 9

Pichardo, B., Sparke, L. S., \& Aguilar, L. A. 2005, MNRAS, 359, 521

Pichardo, B., Sparke, L. S., \& Aguilar, L. A. 2008, MNRAS, 391, 815

Piétu, V., Dutrey, A., Guilloteau, S., Chapillon, E., \& Pety, J. 2006, A\&A, 460, L43

Prato, L., Huerta, M., Johns-Krull, C. M., et al. 2008, ApJ, 687, L103

Przygodda, F., van Boekel, R., Àbrahàm, P., et al. 2003, A\&A, 412, L43

Queloz, D., Henry, G. W., Sivan, J. P., et al. 2001, A\&A, 379, 279

Reipurth, B., Lindgren, H., Mayor, M., Mermilliod, J.-C., \& Cramer, N. 2002, AJ, 124, 2813

Rice, W. K. M., Armitage, P. J., Wood, K., \& Lodato, G. 2006, MNRAS, 373, 1619

Rigliaco, E., Natta, A., Randich, S., Testi, L., \& Biazzo, K. 2011, A\&A, 525, A47

Rigliaco, E., Natta, A., Testi, L., et al. 2012, A\&A, 548, A56

Roccatagliata, V., Ratzka, T., Henning, T., et al. 2011, A\&A, 534, A33

Romanova, M. M., Ustyugova, G. V., Koldoba, A. V., \& Lovelace, R. V. E. 2004, ApJ, 610, 920

Rosenfeld, K. A., Andrews, S. M., Wilner, D. J., Kastner, J. H., \& McClure, M. K. 2013, ApJ, 775, 136

Rydgren, A. E., \& Vrba, F. J. 1983, AJ, 88, 1017 
Schaller, G., Schaerer, D., Meynet, G., \& Maeder, A. 1992, A\&AS, 96, 269 Schegerer, A. A., Wolf, S., Hummel, C. A., Quanz, S. P., \& Richichi, A. 2009, A\&A, 502, 367

Schlafly, E. F., \& Finkbeiner, D. P. 2011, ApJ, 737, 103

Shevchenko, V. S., Grankin, K. N., Mel'Nikov, S. Y., \& Lamzin, S. A. 1998, Astron. Lett., 24, 528

Shu, F. H. 1977, ApJ, 214, 488

Sicilia-Aguilar, A., Hartmann, L., Calvet, N., et al. 2006, ApJ, 638, 897

Sicilia-Aguilar, A., Henning, T., \& Hartmann, L. W. 2010, ApJ, 710, 597

Sicilia-Aguilar, A., Kóspál, Á., Setiawan, J., et al. 2012, A\&A, 544, A93

Siess, L., Dufour, E., \& Forestini, M. 2000, A\&A, 358, 593

Skrutskie, M. F., Cutri, R. M., Stiening, R., et al. 2006, AJ, 131, 1163

Stempels, H. C., \& Gahm, G. F. 2004, A\&A, 421, 1159

Storzer, H., \& Hollenbach, D. 1998, ApJ, 502, L71

Strom, K. M., Strom, S. E., Edwards, S., Cabrit, S., \& Skrutskie, M. F. 1989, AJ, 97, 1451

Stumpff, P. 1980, A\&AS, 41, 1
Tamazian, V. S., Docobo, J. A., White, R. J., \& Woitas, J. 2002, ApJ, 578, 925

Tatulli, E., Isella, A., Natta, A., et al. 2007, A\&A, 464, 55

Tognelli, E., Prada Moroni, P. G., \& Degl'Innocenti, S. 2011, A\&A, 533, A109

Toomre, A. 1964, ApJ, 139, 1217

Torres, G., Stefanik, R. P., Latham, D. W., \& Mazeh, T. 1995, ApJ, 452, 870

van der Marel, N., van Dishoeck, E. F., Bruderer, S., et al. 2013, Science, 340 1199

Wang, Sharon, X., Wright, J. T., Cochran, W., et al. 2012, ApJ, 761, 46

Weise, P., Launhardt, R., Setiawan, J., \& Henning, T. 2010, A\&A, 517, A88

White, R. J., \& Ghez, A. M. 2001, ApJ, 556, 265

White, R. J., \& Hillenbrand, L. A. 2005, ApJ, 621, L65

White, R. J., Ghez, A. M., Reid, I. N., \& Schultz, G. 1999, ApJ, 520, 811

Wright, E. L., Eisenhardt, P. R. M., Mainzer, A. K., et al. 2010, AJ, 140, 1868

Wright, J. T., \& Howard, A. W. 2009, ApJS, 182, 205

Zechmeister, M., \& Kürster, M. 2009, A\&A, 496, 577

Zhu, Z., Nelson, R. P., Dong, R., Espaillat, C., \& Hartmann, L. 2012, ApJ, 755, 
Table 4. EWs ( $\mathrm{A})$ of emission lines and Li absoprtion line of GW Ori.

\begin{tabular}{|c|c|c|c|c|c|c|c|c|c|c|c|c|c|c|}
\hline ID & $\begin{array}{c}\mathrm{H} \gamma \\
(4341 \AA\end{array}$ & $\mathrm{H} \beta$ & $\begin{array}{r}\mathrm{H} \alpha \\
6563\end{array}$ & $\begin{array}{r}\mathrm{He} \mathrm{I} \\
5876 \AA\end{array}$ & $\begin{array}{c}\text { O I } \\
300 \AA\end{array}$ & $\begin{array}{c}\text { O I } \\
363\end{array}$ & $\begin{array}{r}\text { O I } \\
7773\end{array}$ & $\begin{array}{r}\text { O I } \\
446\end{array}$ & $\begin{array}{r}\mathrm{Ca} \text { II } \\
(3933 \AA\end{array}$ & $\begin{array}{r}\mathrm{Ca} \text { II } \\
3968 \AA\end{array}$ & $\begin{array}{c}\mathrm{Ca} \text { II } \\
8498 \AA\end{array}$ & $\begin{array}{c}\text { Ca II } \\
8662 \AA\end{array}$ & $\begin{array}{c}\mathrm{Na} \text { I } \\
90 / 5896\end{array}$ & $\begin{array}{c}\text { Li I } \\
6708 \AA)\end{array}$ \\
\hline 1 & -1.93 & -4.63 & -30.29 & -0.18 & -0.28 & -0.06 & -0.29 & -0.77 & -10.21 & -7.08 & -6.09 & -6.46 & -1.15 & 0.20 \\
\hline 2 & -1.90 & -4.33 & -28.93 & -0.22 & -0.29 & -0.07 & -0.37 & -0.74 & -8.86 & -6.81 & -5.29 & -5.69 & -1.01 & 0.20 \\
\hline 3 & -1.72 & -3.73 & -24.21 & -0.25 & -0.29 & -0.07 & -0.27 & -0.61 & -8.45 & -6.34 & -5.00 & -5.36 & -0.75 & 0.21 \\
\hline 4 & -1.26 & -2.96 & -20.27 & -0.09 & -0.26 & -0.07 & -0.01 & -0.35 & -6.55 & -4.71 & -3.12 & -3.37 & -0.28 & 0.23 \\
\hline 5 & -1.22 & -3.00 & -20.50 & -0.10 & -0.27 & -0.06 & -0.11 & -0.40 & -6.57 & -4.77 & -3.40 & -3.66 & -0.43 & 0.23 \\
\hline 6 & -1.24 & -3.01 & -20.28 & -0.11 & -0.26 & -0.06 & -0.10 & -0.41 & -6.55 & -4.82 & -3.31 & -3.61 & -0.48 & 0.22 \\
\hline 7 & -1.43 & -3.45 & -22.99 & -0.13 & -0.27 & -0.06 & -0.14 & -0.50 & -7.46 & -4.99 & -3.87 & -4.14 & -0.60 & 0.23 \\
\hline 8 & -1.56 & -3.66 & -23.55 & -0.15 & -0.27 & -0.06 & -0.13 & -0.44 & -7.06 & -5.10 & -3.60 & -3.88 & -0.57 & 0.22 \\
\hline 9 & -1.55 & -3.54 & -23.34 & -0.23 & -0.26 & -0.06 & -0.13 & -0.51 & -7.84 & -5.35 & -4.00 & -4.30 & -0.56 & 0.22 \\
\hline 10 & -1.26 & -3.12 & -22.21 & -0.08 & -0.26 & -0.05 & -0.06 & -0.43 & -6.48 & -4.53 & -3.21 & -3.60 & -0.50 & 0.23 \\
\hline 11 & -1.22 & -2.92 & -20.61 & -0.08 & -0.25 & -0.06 & $\ldots$ & $\ldots$ & -7.77 & -5.16 & $\ldots$ & $\ldots$ & -0.36 & 0.20 \\
\hline 12 & -1.45 & -3.36 & -22.28 & -0.14 & -0.25 & -0.06 & $\ldots$ & $\ldots$ & -8.06 & -5.53 & $\ldots$ & $\ldots$ & -0.52 & 0.22 \\
\hline 13 & -1.43 & -3.38 & -22.41 & -0.16 & -0.25 & -0.06 & $\ldots$ & $\ldots$ & -8.19 & -5.42 & $\ldots$ & $\ldots$ & -0.40 & 0.21 \\
\hline 14 & -1.26 & -2.93 & -19.07 & -0.09 & -0.27 & -0.07 & -0.00 & -0.33 & -6.54 & -4.87 & -3.26 & -3.50 & -0.37 & 0.22 \\
\hline 15 & -1.24 & -2.85 & -18.89 & -0.07 & -0.27 & -0.07 & -0.03 & -0.36 & -6.87 & -4.74 & -3.27 & -3.53 & -0.46 & 0.22 \\
\hline 16 & -1.20 & -2.73 & -18.01 & -0.06 & -0.26 & -0.07 & -0.04 & -0.39 & -6.15 & -4.47 & -2.97 & -3.27 & -0.41 & 0.22 \\
\hline 17 & -1.12 & -2.64 & -18.00 & -0.07 & -0.27 & -0.07 & -0.03 & -0.36 & -6.03 & -4.65 & -2.93 & -3.22 & -0.44 & 0.22 \\
\hline 18 & -1.24 & -2.81 & -19.35 & -0.14 & -0.27 & -0.07 & -0.10 & -0.39 & -6.27 & -4.37 & -3.08 & -3.29 & -0.49 & 0.22 \\
\hline 19 & -1.18 & -2.85 & -19.04 & -0.15 & -0.27 & -0.06 & -0.09 & -0.31 & -6.28 & -4.63 & -3.09 & -3.28 & -0.48 & 0.22 \\
\hline 20 & -1.20 & -2.84 & -18.69 & -0.09 & -0.28 & -0.06 & -0.04 & -0.37 & -6.92 & -4.79 & -3.30 & -3.46 & -0.52 & 0.22 \\
\hline 21 & -1.30 & -3.01 & -19.35 & -0.08 & -0.27 & -0.06 & -0.04 & -0.35 & -7.05 & -4.78 & -3.26 & -3.51 & -0.50 & 0.23 \\
\hline 22 & -1.19 & -2.86 & -18.65 & -0.13 & -0.26 & -0.08 & -0.03 & -0.33 & -6.48 & -4.69 & -2.97 & -3.22 & -0.50 & 0.22 \\
\hline 23 & -1.25 & -2.88 & -18.84 & -0.14 & -0.27 & -0.06 & -0.05 & -0.32 & -6.56 & -4.73 & -3.03 & -3.33 & -0.50 & 0.22 \\
\hline 24 & -1.48 & -3.17 & -20.43 & -0.19 & -0.27 & -0.07 & -0.12 & -0.41 & -7.00 & -4 & -3.31 & -3.58 & -0.50 & 0.23 \\
\hline 25 & -1.44 & -3.20 & -20.57 & -0.19 & -0.27 & -0.07 & -0.16 & -0.39 & -7.04 & -4.97 & -3.36 & -3.60 & -0.51 & 0.22 \\
\hline 26 & -1.15 & -2.68 & -19.15 & -0.09 & -0.27 & -0.07 & -0.01 & -0.35 & -6.21 & -4.54 & -2.98 & -3.17 & -0.47 & 0.23 \\
\hline 27 & -1.17 & -2.73 & -19.06 & -0.07 & -0.26 & -0.07 & -0.01 & -0.33 & -6 & -4 & -3.01 & -3.22 & -0.47 & 0.24 \\
\hline 28 & -1.93 & -4.21 & -24.20 & -0.22 & -0.26 & -0.07 & -0.21 & -0.65 & -8.44 & -5.92 & -4.39 & -4.67 & -0.70 & 0.22 \\
\hline 29 & -1.84 & -4.04 & -23.82 & -0.22 & -0.27 & -0.07 & -0.19 & -0.53 & -7.93 & -6.00 & -4.32 & -4.62 & -0.77 & 0.22 \\
\hline 30 & -1.52 & -3.44 & -21.68 & -0.19 & -0.26 & -0.07 & -0.10 & -0.50 & -7.37 & -5.17 & -3.59 & -3.96 & -0.53 & 0.22 \\
\hline 31 & -1.42 & -3.30 & -21.36 & -0.18 & -0.27 & -0.08 & -0.10 & -0.42 & -7.08 & -5.02 & -3.45 & -3.78 & -0.52 & 0.23 \\
\hline 32 & -1.41 & -3.23 & -21.43 & -0.10 & -0.28 & -0.07 & -0.08 & -0.44 & -6.66 & -5.07 & -3.40 & -3.73 & -0.52 & 0.23 \\
\hline 33 & -1.69 & -3.53 & -22.86 & -0.28 & -0.30 & -0.07 & $\ldots$ & $\ldots$ & -8.32 & -5.85 & $\ldots$ & $\ldots$ & -0.75 & 0.24 \\
\hline 34 & -1.01 & -2.25 & -19.46 & -0.18 & -0.32 & -0.07 & $\ldots$ & $\ldots$ & -8.08 & -5.14 & $\ldots$ & $\ldots$ & -0.60 & 0.21 \\
\hline 35 & -1.52 & -3.41 & -21.98 & -0.24 & -0.30 & -0.09 & $\ldots$ & $\ldots$ & -8.70 & -5.60 & $\ldots$ & $\ldots$ & -0.54 & 0.23 \\
\hline 36 & -1.02 & -2.31 & -19.10 & -0.17 & -0.34 & -0.07 & $\ldots$ & $\ldots$ & -8.02 & -5.21 & $\ldots$ & $\ldots$ & -0.42 & 0.21 \\
\hline 37 & -1.16 & -3.21 & -19.67 & -0.08 & -0.31 & -0.07 & $\ldots$ & $\ldots$ & -6.53 & -4.41 & $\ldots$ & $\ldots$ & -0.45 & 0.23 \\
\hline 38 & -1.25 & -3.24 & -21.94 & -0.09 & -0.27 & -0.07 & $\ldots$ & $\ldots$ & -7.54 & -4.90 & $\ldots$ & $\ldots$ & -0.53 & 0.21 \\
\hline 39 & -1.45 & -3.47 & -23.33 & -0.18 & -0.30 & -0.07 & $\ldots$ & $\ldots$ & -7.47 & -4.90 & $\cdots$ & $\ldots$ & -0.55 & 0.23 \\
\hline 40 & -1.32 & -3.35 & -22.39 & -0.13 & -0.30 & -0.08 & $\ldots$ & & -7.74 & -5.18 & $\ldots$ & $\ldots$ & -0.59 & 0.22 \\
\hline 41 & -1.79 & -4.28 & -28.70 & -0.15 & -0.34 & -0.11 & -0.27 & -0.69 & -6.86 & -5.20 & -4.76 & -5.15 & -0.92 & 0.22 \\
\hline 42 & -1.39 & -4.41 & -31.58 & -0.13 & -0.37 & -0.21 & -0.02 & -0.40 & $\ldots$ & -1.70 & -4.00 & -3.98 & -0.52 & 0.24 \\
\hline 43 & -1.83 & -4.32 & -31.13 & -0.17 & -0.34 & -0.11 & -0.16 & -0.64 & -7.80 & -5.85 & -4.34 & -4.64 & -0.81 & 0.22 \\
\hline 44 & -1.47 & -3.46 & -27.38 & -0.08 & -0.35 & -0.09 & 0.03 & -0.45 & -6.22 & -3.78 & -3.07 & -3.42 & -0.50 & 0.21 \\
\hline 45 & -1.75 & -4.47 & -30.98 & -0.14 & -0.35 & -0.11 & -0.13 & -0.63 & -8.07 & -5.54 & -4.85 & -4.92 & -0.76 & 0.22 \\
\hline 46 & -1.63 & -4.02 & -27.78 & -0.11 & -0.36 & -0.11 & 0.03 & -0.46 & -7.32 & -5.42 & -3.69 & -3.88 & -0.56 & 0.23 \\
\hline 47 & -1.71 & -4.19 & -27.03 & -0.17 & -0.35 & -0.10 & -0.07 & -0.54 & -7.88 & -5.30 & -4.15 & -4.29 & -0.70 & 0.23 \\
\hline 48 & -1.40 & -3.65 & -26.44 & -0.07 & -0.35 & -0.09 & -0.07 & -0.49 & -6.95 & -5.10 & -3.55 & -3.80 & -0.68 & 0.22 \\
\hline 49 & -1.29 & -3.07 & -23.84 & -0.09 & -0.34 & -0.09 & -0.11 & -0.48 & -6.47 & -4.95 & -3.59 & -3.83 & -0.48 & 0.23 \\
\hline 50 & -1.25 & -2.99 & -22.36 & -0.06 & -0.33 & -0.09 & -0.06 & -0.43 & -5.89 & -4.40 & -3.28 & -3.43 & -0.43 & 0.23 \\
\hline 51 & -1.67 & -3.88 & -25.78 & -0.21 & -0.33 & -0.07 & -0.16 & -0.51 & -7.25 & -5.27 & -3.44 & -3.84 & -0.51 & 0.21 \\
\hline 52 & -1.71 & -3.90 & -25.54 & -0.17 & -0.33 & -0.11 & -0.13 & -0.46 & -7.00 & -5.29 & -3.47 & -3.90 & -0.49 & 0.23 \\
\hline 53 & -1.54 & -3.55 & -25.24 & -0.11 & -0.32 & -0.09 & -0.05 & -0.42 & -6.42 & -4.57 & -3.19 & -3.48 & -0.45 & 0.24 \\
\hline 54 & -1.18 & -2.80 & -22.46 & -0.05 & -0.32 & -0.09 & -0.03 & -0.38 & -5.74 & -4.22 & -2.85 & -3.16 & -0.37 & 0.23 \\
\hline 55 & -1.42 & -3.55 & -27.59 & -0.11 & -0.32 & -0.11 & 0.12 & -0.42 & -6.15 & -4.98 & -3.73 & -3.59 & -0.58 & 0.23 \\
\hline 56 & -1.32 & -3.37 & -26.22 & -0.08 & -0.32 & -0.09 & -0.03 & -0.39 & -6.98 & -4.80 & -3.35 & -3.66 & -0.47 & 0.24 \\
\hline 57 & -1.23 & -3.02 & -23.09 & -0.02 & -0.29 & -0.09 & -0.04 & -0.39 & -5.31 & -4.21 & -3.15 & -3.43 & -0.47 & 0.24 \\
\hline 58 & -1.28 & -3.08 & -23.15 & -0.05 & -0.32 & -0.11 & -0.01 & -0.36 & -5.83 & -4.57 & -3.37 & -3.48 & -0.48 & 0.23 \\
\hline
\end{tabular}

